\title{
The Schrödinger operator with Morse potential on the right half-line
}

\author{
JEFFREY C. LAGARIAS
}

\begin{abstract}
This paper studies the Schrödinger operator with Morse potential $V_{k}(u)=\frac{1}{4} \mathrm{e}^{2 u}+k \mathrm{e}^{u}$ on a right half-line $\left[u_{0}, \infty\right)$, and determines the Weyl asymptotics of eigenvalues for constant boundary conditions at the endpoint $u_{0}$. In consequence, it obtains information on the location of zeros of the Whittaker function $W_{\kappa, \mu}(x)$, for fixed real parameters $\kappa, x$ with $x>0$, viewed as an entire function of the complex variable $\mu$. In this case all zeros lie on the imaginary axis, with the possible exception, if $\kappa>0$ of a finite number of real zeros which lie in the interval $-\kappa<\mu<\kappa$. We obtain an asymptotic formula for number of zeros $N(T)=\left\{\rho\left|W_{\kappa, \rho}(x)=0,\right| \operatorname{Im}(\rho) \mid<T\right\}$ of the form $N(T)=\frac{2}{\pi} T \log T+\frac{2}{\pi}(2 \log 2-1-\log x) T+O(1)$. Parallels are observed with zeros of the Riemann zeta function.
\end{abstract}

\section{Introduction}

The one-dimensional potential function $V_{A, B}(u)=A \mathrm{e}^{2 u}+B \mathrm{e}^{u}$ with $A>0$ and $B$ real was proposed in 1929 by Philip Morse [50] as an approximation to the (radial) quantum-mechanical potential for diatomic molecules, cf. [30, Problem 70]. From a quantum physics viewpoint this corresponds to the Schrödinger operator

$$
-\frac{d^{2}}{d u^{2}}+V_{A, B}(u)
$$

viewed either on the line $(-\infty, \infty)$ or on the left half-line $\left(-\infty, u_{0}\right]$, with a suitable boundary condition imposed at the right endpoint $u_{0}$. By rescaling the independent variable we may, without loss of generality, reduce to the case $A=\frac{1}{4}$, and in that case relabel the other parameter, setting $B=k$, obtaining the potential $V_{k}(u)=\frac{1}{4} \mathrm{e}^{2 u}+k \mathrm{e}^{u}$. The Morse potential on the line, with $k=0$, arises elsewhere in physics in connection with the Liouville model on the hyperbolic plane [34] and in describing motion on the hyperbolic plane with a magnetic field [35]. The latter authors also relate it to the Selberg trace formula on compact hyperbolic surfaces. 
In both the line and left half-line cases the Schrödinger operator has an absolutely continuous spectrum of multiplicity one supported on the half-line $[0, \infty)$, plus a finite (possibly empty) discrete spectrum, lying strictly below the absolutely continuous spectrum. On the line, the operator is essentially self-adjoint for the standard domain of smooth rapidly decreasing functions, and is exactly solvable. Formulas for its resolvent (Green's function) have been obtained, e.g., [35, Proposition 4.1(i)]. The absolutely continuous spectrum is described using generalized eigenfunctions given in terms of Whittaker $M_{\kappa, \mu}(x)$-functions, and the discrete spectrum has eigenfunctions given using rescaled Laguerre polynomials, cf. [36].

\subsection{Morse potential on right half-line}

We treat the Morse potential $V_{k}(u)$ on the right half-line $\left[u_{0}, \infty\right)$, with constant boundary conditions imposed at the endpoint $u_{0}$. The spectrum of this operator is quite different from the cases above: it is pure discrete, simple and bounded below. This follows because the Morse potential is bounded below and is unbounded as $u \rightarrow \infty$. The discrete eigenfunctions on the halfline are expressible in terms of Whittaker functions $W_{\kappa, \mu}(x)$, in which $\mu$ is the eigenvalue parameter. We consider

$$
\left(-\frac{d^{2}}{d u^{2}}+V_{k}(u)\right) \psi(u)=E \psi(u),
$$

and in Section 2 we observe for all complex $E$ there is a unique eigenfunction (up to scaling) in $L^{2}\left(\left[u_{0}, \infty\right), d u\right.$ ) with eigenvalue $E=-\mu^{2}$ given by

$$
\psi(u, E):=\mathrm{e}^{-\frac{1}{2} u} W_{-k, \pm \mu}\left(\mathrm{e}^{u}\right) .
$$

The function $\psi(u, E)$ is well-defined since $W_{k, \mu}(x)=W_{k,-\mu}(x)$ for all $\mu \in \mathbb{C}$, when $k$ is real and $x=\mathrm{e}^{u}$ is positive real. The allowed real eigenvalues $E_{0}<E_{1}<E_{2}<\cdots$ in the discrete spectrum are selected by the boundary conditions at the left endpoint $u_{0}$, with corresponding value $x_{0}=\mathrm{e}^{u_{0}}$, since we are in the limit point case at the singular endpoint $u=+\infty$.

This paper determines the Weyl asymptotics of the spectrum for fixed constant boundary conditions, obtaining in Section 4 that

$$
\#\left\{E_{n} \leq T\right\}=c_{1} \sqrt{T} \log T+c_{2} \sqrt{T}+O(1)
$$

as $T \rightarrow \infty$. Dirichlet boundary conditions correspond to zeros of the Whittaker function in the $\mu$-variable, holding the other variables fixed. The results 
above, applied with these boundary conditions, lead to determination of the location and asymptotic distribution of the zeros of these Whittaker functions, given in Section 5 below. Namely, these zeros all lie on the imaginary axis, with finitely many exceptions, with asymptotics given in (1.3) below. The exceptional zeros exist only if $\kappa=-k>0$, and then all lie on the real axis in the open interval $(-|k|,|k|)$.

\subsection{Analogy with distribution of Riemann zeta zeros}

Our main interest in this family of operators arose from number theory, to view them as "toy models" for operators whose eigenvalues represent zeros of the Riemann zeta function. We note there has been a persistent effort to find a spectral interpretation of the Riemann zeta zeros. In this regard, by a "Hilbert-Polya" operator we will mean an (unbounded) self-adjoint operator on a Hilbert space whose spectrum encodes the zeta zeros, such that the self-adjointness of the operator encodes the Riemann hypothesis. Here we consider one-dimensional Schrödinger operators on a half-line from this viewpoint.

To compare the distribution of Morse half-line eigenvalues with zeros of the Riemann zeta function $\zeta(s)$, in our context the $s$-variable in $\zeta(s)$ corresponds to the $\mu$-variable in the Whittaker function $W_{\kappa, \mu}(x)$ via $s:=$ $\mu-\frac{1}{2}$; this variable change maps the imaginary axis in the $\mu$-variable to the critical line $\operatorname{Re}(s)=\frac{1}{2}$ in the $s$-variable. One point of this paper is that the function

$$
Z_{1}(s):=Z_{1}\left(s ; \kappa, x_{0}\right)=W_{\kappa, s-\frac{1}{2}}\left(x_{0}\right)
$$

for fixed real $\kappa$ and fixed real $x_{0}>0$ has a number of properties analogous to the Riemann $\xi$-function

$$
\xi(s)=\frac{1}{2} s(s-1) \pi^{-\frac{s}{2}} \Gamma\left(\frac{s}{2}\right) \zeta(s)
$$

Namely, in Theorem 4.1 we establish the following properties of $Z_{1}(s)$.

(1) $Z_{1}(s)$ is an entire function of order 1 and maximal type, which is real on the real axis and is real on the line $\operatorname{Re}(s)=\frac{1}{2}$.

(2) $Z_{1}(s)$ satisfies the functional equation

$$
Z_{1}(s)=Z_{1}(1-s)
$$


(3) The number $N_{T}^{ \pm}\left(Z_{1}(s)\right)$ of zeros $\rho$ of $Z_{1}(s)$ with $|\rho|<T$ is given as $T \rightarrow \infty$ by

$$
N_{T}^{ \pm}\left(Z_{1}(s)\right)=\frac{2}{\pi} T \log T+\frac{2}{\pi}\left(2 \log 2-1-\log x_{0}\right) T+O(1) .
$$

(4) All but finitely many of the zeros of $Z_{1}(s)$ lie on the line $\operatorname{Re}(s)=\frac{1}{2}$. The finite set of "exceptional zeros" all lie on the real axis, in the open interval $\left(\frac{1}{2}-|\kappa|, \frac{1}{2}+|\kappa|\right)$. There are no exceptional zeros if $\kappa \leq 0$.

(5) All zeros of $Z_{1}(s)$ are simple zeros, except for a possible double zero at $s=\frac{1}{2}$.

The zeros $\rho=\frac{1}{2}+\mathrm{i} \gamma$ of $Z_{1}(s)=Z_{1}\left(s ; \kappa, x_{0}\right)$ have the following spectral interpretation: $\rho$ is a zero of $Z_{1}\left(s ; \kappa, x_{0}\right)$ if and only if $E=\gamma^{2}$ is an eigenvalue of the Schrödinger operator for the Morse potential $V_{k}(u)$ on the half-line $\left[u_{0}, \infty\right)$ for $k=-\kappa$ and $x_{0}=\mathrm{e}^{u_{0}}$, with Dirichlet boundary conditions taken at the left endpoint $u_{0}$. The self-adjointness of this problem leads to the "modified Riemann hypothesis" property (4). This spectrum is bounded below by $E>-\kappa^{2}$, and the eigenvalues $E<0$ correspond to exceptional zeros of $Z_{1}\left(s ; \kappa, x_{0}\right)$ lying on the real axis.

In comparison with $Z_{1}(s)$, the Riemann $\xi$-function $\xi(s)$ has properties (1) and (2). Analogous to property (3), the number of zeros $N_{T}^{ \pm}(\xi(s))$ of the Riemann $\xi$-function with $|\rho|<T$ satisfies

$$
N_{T}^{ \pm}(\xi(s))=\frac{1}{\pi} T \log T+\frac{1}{\pi}(-\log 2 \pi-1) T+O(\log T),
$$

cf. [26, Section 6.7] or [68, Theorem 9.4]. Analogues of properties (4) and (5) are conjectured to hold for $\xi(s)$. Property (4) is a "modified Riemann hypothesis", which permits a finite number of exceptional zeros to occur on the real axis. The Riemann $\xi$-function has no exceptional zeros, but the possibility of exceptional zeros remains open for various generalizations of the Riemann zeta function, e.g. automorphic $L$-functions ([37]). Concerning property (5), the zeta function is reported to have simple zeros for the first $10^{13}$ zeros on the critical line.

A possible weakness of the Morse potential as a "toy model" for a spectral interpretation of the zeta zeros is that its eigenvalue distribution only reproduces the main terms in the asymptotic number of zeros, it does not correctly model the "lower order" asymptotic behavior of zeta zeros. The detailed structure of scaled zeta zeros is conjectured to be described by the GUE distribution of random matrix theory, and the functions here do not reproduce this behavior. This detailed behavior presumably encodes all 
the number theory having to do with the distribution of prime numbers. This is discussed further in Section 5. A putative "Hilbert-Polya" operator Schrödinger potential, if it exists, will likely have exotic features to produce such features in its spectrum.

\subsection{Schrödinger operators and de Branges spaces as spectral models for zeta zeros}

Recently we observed that, provided the Riemann hypothesis holds, there is a natural candidate for a "Hilbert-Polya" operator within the framework of the de Branges theory of Hilbert spaces of entire functions [41, 42]. The de Branges theory provides model operators for a certain class of operators, which includes generalizations of operators from singular Sturm-Liouville problems of the type considered here. This theory gives a representation of the associated operator as a (self-adjoint) canonical system of differential equations (defined in Section 5), in which each zeta zero $\rho=\frac{1}{2}+\mathrm{i} \gamma$ corresponds to a simple real eigenvalue $\gamma$ of the resulting canonical system, so that the operator has a simple spectrum that is unbounded above and below. The results in $[41,42]$ show that if appropriate Riemann hypotheses hold then the associated canonical systems must exist. We call these Hilbert-Polya canonical systems.

The connection to Schrödinger operators is that, in favorable circumstances, a canonical system may be formally transformed to a Schrödinger operator on a half-line. This transform squares the eigenvalues, so that the resulting Schrödinger spectrum is bounded below. If this transformation could legitimately be done for the putative "Hilbert-Polya" de Branges spaces above, then it would result in a "Hilbert-Polya" operator that is a Schrödinger operator on a half-line. If so, it would be very interesting to determine the associated potential of this operator. Note that this "HilbertPolya" potential, if it exists at all, may be highly singular, involve fractal features, and be describable only as a distribution. We remark that additional symmetries of the problem indicate that this Schrödinger operator necessarily would impose Dirichlet boundary conditions at the left endpoint.

The Schrödinger operators treated in this paper may provide hints about features of this possible "Hilbert-Polya" potential. In particular, they give the dependence of eigenvalue asymptotics on the endpoint of the interval. Furthermore, even if such a "Hilbert-Polya" Schrödinger operator does not exist, these examples may still give hints about the canonical system of the de Branges space associated to RH. Namely, there exists a de Branges space with explicitly known canonical system for which the nonlinear transform 
above can be carried out to give a "toy model" Morse potential Schrödinger operator with $k=-\frac{1}{2}([43])$. The form of this particular canonical system then gives hints about features of the associated canonical system coefficients of the Hilbert-Polya canonical system, which is guaranteed to exist if RH holds.

\subsection{Previous work}

There has been a long history of work on special functions whose zeros mimic the zeros of the Riemann zeta function, some of them associated to differential equations. Polya [53] observed in 1926 that the zeros of the $K$-Bessel function $K_{\mu}(x)$ for fixed $x>0$ lie on the imaginary axis and have asymptotics similar to that of the Riemann zeta function. In fact Polya actually studies a function given by an integral representation, which in retrospect was shown to be a $K$-Bessel function, cf. [31]. In 1927 Polya [54] gave a second proof of the property that these zeros lie on the imaginary axis which made use of a second-order differential equation in an auxiliary variable. Another "toy model" discussed by Polya is described in [68, Section 10.1]. It involves a shifted sum of two $K$-Bessel functions, discussed more recently in [31]. The present work can be viewed as obtaining an extension to a one-parameter family of Polya's results, in the sense that a $K$-Bessel function is essentially a special case of the Whittaker functions considered here, namely $K_{\mu}(w)=\sqrt{\frac{\pi}{2 w}} W_{0, \mu}(2 w)$. Recent work of Biane [5] notes an operator-theoretic parallel between the $K$-Bessel function and the Riemann $\xi$-function, and makes a very interesting new connection between these two functions in terms of probability theory.

Aside from the "toy model" aspect, our results provide some new information on the location of zeros of Whittaker functions. Here there has been considerable previous work locating zeros of $W_{\kappa, \mu}(z)$, in connection with various applied problems. This work mostly considers the case of fixed $\kappa$ and $\mu$, and varies the (complex) variable $z$. In 1915 Milne [49] studied real $\kappa$ and $\mu$ and determined the nature of zeros in the $z$-variable on the positive real axis in special cases. In 1941 Tvestkoff [71] announced results on the location of all zeros with $\kappa$ and $\mu$ real and $z=x>0$ a positive real, and in 1950 Tricomi [69] gave detailed proofs. Their work determines the locations of the finite set of "exceptional zeros" in (4) above. In 1960 Dikii [21] and Dyson [25] independently considered the case of $\kappa$ real, $\mu$ purely imaginary, and $z$ a (nonzero) complex variable. Here $W_{\kappa, \mu}(z)$ is multi-valued in the $z$-variable with a singularity at $z=0$, and the $z$-plane is cut along the nonpositive real axis. Their work arose from analysis of the stability of a hydrodynamic model of an incompressible atmosphere having density 
decreasing exponentially with height, with wind velocity increasing linearly with height, see [22] and [16], respectively. Both Dikii and Dyson showed that all the zeros in the complex variable $z$ in the region $-\pi \leq \arg (z) \leq \pi$ lie on the positive real axis, and that there are infinitely many such zeros, having the singular point $z=0$ as a limit point. The results here complement these analyses by determining the location and density of zeros when $\kappa$ is real and $z$ is positive real.

Our interest in de Branges spaces was influenced by recent work of Burnol $[10,11,13]$, which described a scattering associated to the Fourier and Hankel transforms, and pointed out a de Branges space interpretation. Burnol constructed certain Dirac operators and Schrödinger operators (corresponding to particular canonical systems) which have eigenvalue asymptotics of the general form $c T \log T+O(T)$. In these papers Burnol did not explicitly note these asymptotics, but he elsewhere derived similar eigenvalue asymptotics for related operators [12].

\subsection{Methods}

The proofs given here are entirely classical, within the framework of Titchmarsh [67]. They break no new ground methodologically. The main point of this paper is rather to explain an analogy of these operators with a "HilbertPolya" operator for the Riemann zeta zeros. In particular, we explicitly determine the eigenvalue asymptotics (1.3), with all details included, for the convenience of number theorists.

\subsection{Contents of paper}

The detailed contents of this paper are as follows.

In Section 2 we determine the $L^{2}$-eigenfunctions of the Schrödinger operator with the Morse potential on the half-line $\left[u_{0}, \infty\right)$, and determine its solutions for constant boundary conditions at the left endpoint $u_{0}$, particularly Dirichlet boundary conditions (Theorem 2.1). This is a singular boundary value problem. Using Sturm-Liouville theory we deduce certain monotonicity properties of the eigenvalues as functions of the parameters (Theorem 2.2).

In Section 3 we show that the density of eigenvalues $\#\{E \leq T\}$ of the Schrödinger operator is asymptotic to $c_{1} \sqrt{T} \log T+c_{2} \sqrt{T}+O(1)$, for certain constants $c_{1}, c_{2}$ (Theorem 3.2).

In Section 4, we specialize to Dirichlet boundary conditions, and apply these results to Whittaker functions $W_{\kappa, \mu}(x)$, viewed as a function of the 
complex variable $\mu$. We establish that the zeros of $Z(\mu):=W_{\kappa, \mu}(x)$ lie on the imaginary $\mu$-axis, with finitely many exceptions, which themselves lie on the real axis, and we determine their asymptotic density to height $T$ to be given by (1.3) above (Theorem 4.1).

In Section 5 we discuss analogies of these results with zeta functions in number theory and with de Branges spaces. In this connection, it is well known that one can relate de Branges spaces to Schrödinger operators (Sturm-Liouville operators) in various ways, see [23, 24,57]. Specific de Branges spaces having number theory content were related to Schrödinger and Dirac operators by Burnol [9-11,13,14].

In Section 6 we make some concluding remarks, discussing analytic spectral invariants associated to these operators, the Weyl-Titchmarsh $m$-function of a Schrödinger operator and an analogous quantity in the de Branges theory, which we call the de Branges $m$-function.

There are two appendices. Appendix A reviews properties of Whittaker functions $M_{\kappa, \mu}(z)$ and $W_{\kappa, \mu}(z)$ as functions of three complex variables $(\kappa, \mu, z)$. Appendix B gives formulas for the principal Weyl-Titchmarsh $m$-functions associated to Morse potentials $V_{k}(u)$ on the right half-line. This function encodes spectral data about the potential, and we show it is a ratio of Whittaker $W$-functions with shifted $\kappa$-parameter values, shifted by a constant. It is well known that a Schrödinger equation potential $V(u)$ can be uniquely reconstructed from the principal Weyl-Titchmarsh $m$-function under general conditions.

Notation. We follow the mathematical convention that Hilbert spaces have scalar products $\langle f, g\rangle$ that are linear in the first factor and conjugatelinear in the second factor, as in $[17,45]$. This is done for compatibility with the theory of de Branges [7, p. 50] discussed in Section 5. References to Reed and Simon [55], who use the physics convention that Hilbert spaces are conjugate linear in the first factor, must be adjusted for this fact. We set $L^{2}\left(u_{0}, \infty\right):=L^{2}\left[\left(u_{0}, \infty\right), d u\right]$. The variables $a, b, c, d, k, t, u, v, x, y$ denote real variables. The variables $z=x+\mathrm{i} y, s=\sigma+\mathrm{i} t, \kappa=a+\mathrm{i} b, \mu=c+\mathrm{i} d$ denote complex variables.

\section{Morse potential on the right half-line}

Let $k$ be an arbitrary real number. We consider the Schrödinger operator $H=-\frac{d^{2}}{d u^{2}}+V(u)$ for the Morse potential

$$
V_{k}(u)=\frac{1}{4} \mathrm{e}^{2 u}+k \mathrm{e}^{u}
$$


The Schrödinger operator with Morse potential on the right half-line 331

on the half-line $\left[u_{0}, \infty\right)$. This is equivalent to treating the more general potential

$$
V_{A, B}(v)=A \mathrm{e}^{2 v}+B \mathrm{e}^{v}
$$

with $A>0$ and $B$ real on the half-line $\left[v_{0}, \infty\right)$, since by a translation of the time variable $v=u-\frac{1}{2} \log \frac{A}{4}$, we reduce to a potential of the form $V_{k}(u)$ above with $k=\frac{2 B}{\sqrt{A}}$ on the half-line $\left[u_{0}, \infty\right)$ with $u_{0}=v_{0}-\frac{1}{2} \log \frac{A}{4}$.

Theorem 2.1. The Schrödinger equation

$$
\left(-\frac{d^{2}}{d u^{2}}+V_{k}(u)\right) \psi(u)=E \psi(u)
$$

for the Morse potential $V_{k}(u)=\frac{1}{4} \mathrm{e}^{2 u}+k \mathrm{e}^{u}$ on a half-line $\left[u_{0}, \infty\right)$ has the following properties.

(1) The two-dimensional complex vector space $\mathcal{E}_{E}\left(V_{k}(u)\right)$ of solutions $\varphi(u)$ is given by

$$
\varphi(u)=\mathrm{e}^{-\frac{u}{2}} f\left(\mathrm{e}^{u}\right)
$$

in which $f(x)$ is any solution to Whittaker's differential equation

$$
\left(\frac{d^{2}}{d x^{2}}+\left(-\frac{1}{4}+\frac{\kappa}{x}+\frac{\frac{1}{4}-\mu^{2}}{x^{2}}\right)\right) f(x)=0,
$$

with parameters $(\kappa, \mu)$ given by $\kappa=-k$ and $\mu^{2}=-E$.

(2) For arbitrary $E \in \mathbb{C}$ there is a one-dimensional subspace of $\mathcal{E}_{E}\left(V_{k}(u)\right)$ of solutions that belong to $L^{2}\left(u_{0}, \infty\right)$. Setting $E=z^{2}$, this subspace is spanned by a solution $\psi(u, E)$ given by

$$
\psi\left(u, z^{2}\right):=\mathrm{e}^{-\frac{u}{2}} W_{-k, \mathrm{i} z}\left(\mathrm{e}^{u}\right)=\mathrm{e}^{-\frac{u}{2}} W_{-k,-\mathrm{i} z}\left(\mathrm{e}^{u}\right),
$$

where $W_{\kappa, \mu}(x)$ denotes the Whittaker $W$-function.

(3) The potential $V_{k}(u)$ is of limit point type at $+\infty$. If the constant boundary conditions

$$
(\cos \alpha) \psi\left(u_{0}\right)+(\sin \alpha) \psi^{\prime}\left(u_{0}\right)=0
$$

for fixed $0 \leq \alpha<2 \pi$ are imposed at the left endpoint $u=u_{0}$, then the Schrödinger equation is self-adjoint. This operator has a pure discrete 
simple real spectrum, bounded below. For Dirichlet boundary conditions for parameter values $k \geq 0$, the smallest eigenvalue $E_{0}>0$; for parameter values $k<0$, one has $E_{0}>-k^{2}$.

Remark. The case $\alpha=0$ (resp. $\alpha=\frac{\pi}{2}$ ) gives Dirichlet (resp. Neumann) boundary conditions at the left endpoint. Recall that for general boundary conditions (2.5), there is no uniform lower bound on the spectrum as $\alpha$ is varied over $0 \leq \alpha \leq 2 \pi$. The eigenvalue $E_{0}=E_{0}(\alpha)$ is a continuous function of $\alpha$ except for a jump discontinuity at $\alpha=0$.

Proof. (1) Whittaker's differential equation (2.3) with parameters $(\kappa, \mu)$ was studied in 1904 by Whittaker [73]. Properties of its solutions are given in [8], [74, Chapter 16]. Note that the parameters $\pm \mu$ give the same solution space, since $\mu$ only enters the differential equation via $\mu^{2}$. For fixed $(\kappa, \mu)$ (2.3) has a two-dimensional complex vector space of solutions $\mathcal{E}_{E}\left(V_{k}(u)\right)$ (for $z$ on the positive real axis). This vector space is spanned by the Whittaker functions $\left(W_{\kappa, \mu}(z), M_{\kappa, \mu}(z)\right)$, except when $\kappa$ is a nonpositive integer. (Another basis is $\left(W_{\kappa,-\mu}(z), M_{\kappa,-\mu}(z)\right.$.) Note that $W_{\kappa, \mu}(z)=W_{\kappa,-\mu}(z)$ but in general $M_{\kappa, \mu}(z) \neq M_{\kappa,-\mu}(z)$. One calculates by direct substitution that if $f(z)$ is any solution to Whittaker's equation (2.3), then $\varphi(u):=\mathrm{e}^{-\frac{u}{2}} f\left(\mathrm{e}^{u}\right)$ satisfies the Schrödinger equation

$$
\left(-\frac{d^{2}}{d u^{2}}+V_{-\kappa}(u)\right) \phi(u)=-\mu^{2} \phi(u) .
$$

Choosing $(\kappa, \mu)=(-k, \pm \sqrt{-E})$, these functions span the two-dimensional space of solutions $\mathcal{E}_{E}\left(V_{-\kappa}(u)\right)$ to the Schrödinger equation (2.1).

(2) The Whittaker function $W_{\kappa, \mu}(z)$ [74, Chapter 16] is a solution $f(z)$ of Whittaker's equation (2.3) which has the property of rapid decay as $z=x \rightarrow$ $\infty$ (on the real axis), made unique by the normalization of its asymptotics

$$
W_{\kappa, \mu}(x)=\mathrm{e}^{-\frac{1}{2} x} x^{\kappa}\left(1+O\left(\frac{1}{x}\right)\right)
$$

as $x \rightarrow \infty$. Viewed in the complex domain, the same asymptotics holds for $|\arg (z)| \leq \pi-\epsilon<\pi$, with the implied constant in the error term depending on $\epsilon>0$. All other linearly independent solutions to Whittaker's differential equation increase exponentially as $x \rightarrow \infty$. Choosing $\kappa=-k$, the particular choice $\varphi(u):=\psi\left(u, z^{2}\right)$ coming from the Whittaker function is the only solution (up to a scalar multiple) that satisfies $\varphi(u) \in L^{2}\left(u_{0}, \infty\right)$ for (any) finite $u_{0}$; it has double-exponential decay as $u \rightarrow \infty$, roughly like $\mathrm{e}^{-\frac{1}{2} \mathrm{e}^{u}}$, as 
specified by the asymptotic formula (2.7). All other solutions have doubleexponential growth as $u \rightarrow \infty$. Solutions (2.4) thus parametrize the subordinate solutions of the Schrödinger operator with Morse potential on the right half-line, in the sense of Gilbert and Pearson [32].

(3) The property that $V_{k}(u)$ is positive for sufficiently large $u$ puts the Schrödinger equation in the limit point case at $u=\infty$ (cf. [55, Theorem X.8]). In particular for each real $E$ there is at most one solution to the Schrödinger equation with eigenvalue $E$ (up to a scalar multiple) that belongs to $L^{2}\left(\left[u_{0}, \infty\right) ; d u\right)$. Here such a solution was exhibited for all complex $E$, given above.

It is known that for continuous potentials $V(u)$ on $\left[u_{0}, \infty\right)$ that are bounded below, the Schrödinger operator $-\frac{d^{2}}{d u^{2}}+V(u)$ with (separated) boundary conditions given by

$$
(\cos \alpha) f\left(u_{0}\right)+(\sin \alpha) f^{\prime}\left(u_{0}\right)=0
$$

for fixed $0 \leq \alpha<2 \pi$ is self-adjoint in $L^{2}\left(\left[u_{0}, \infty\right) ; d u\right)$. To describe the explicit domain of this operator, let $\mathrm{AC}\left[u_{0}, \infty\right]$ denote the set of absolutely continuous functions $f(u)$ on $\left[u_{0}, \infty\right)$ whose (almost everywhere defined) derivative is in $L^{2}\left[u_{0}, \infty\right)$. The associated dense domain for self-adjointness is

$$
\begin{aligned}
\mathcal{D}_{\alpha}:= & \left\{f(u) \in C^{1}\left[u_{0}, \infty\right) \cap L^{2}\left(\left[u_{0}, \infty\right) ; d u\right) \mid f^{\prime}(u) \in \mathrm{AC}\left[u_{0}, \infty\right],\right. \\
& \left.\left(-\frac{d^{2}}{d u^{2}}+V(u)\right) f(u) \in L^{2}\left(\left[u_{0}, \infty\right) ; d u\right), \text { and }(2.8) \text { holds }\right\}
\end{aligned}
$$

cf. [55, p. 144].

The fact that $V_{k}(u)$ is continuous, with $V_{k}(u) \rightarrow \infty$ as $u \rightarrow \infty$ is sufficient to imply that it has pure discrete spectrum, bounded below, for the boundary conditions (2.5), for any choice of $\alpha$ [45, Section 4.1, Lemma 1.2]. This spectrum is simple because for each real $E$ there is at most one solution in $L^{2}\left(\left[u_{0}, \infty\right) ; d u\right)$. The spectrum is bounded below, for any fixed constant boundary conditions, because the potential $V_{k}(u)$ is bounded below.

The Sturm-Liouville theory applies to real potentials having a pure discrete simple spectrum bounded below. It implies that the eigenvalues can be numbered $E_{0}<E_{1}<E_{2}<\cdots$ and the corresponding eigenfunctions $\psi_{0}<\psi_{1}<\psi_{2}<\cdots$ can be chosen real valued. The Sturm-Liouville theory shows that the eigenfunction $\psi_{n}(u)$ has exactly $n$ sign changes on the domain $\left[u_{0}, \infty\right)$, so has $n$ zeros; the bottom eigenfunction $\psi_{0}(u)$ has no changes of sign and can be chosen positive. (See [2, Chapter 8], [17, Chapter 8].)

We now impose Dirichlet boundary conditions $\alpha=0$, and lower bound the minimum eigenvalue $E_{0}$ using the Sturm comparison theorem. For $k \geq 0$ 
the eigenvalues of $V_{k}(u)$ are bounded below by those of $V_{0}(u)$ (with same boundary conditions on the endpoint $\left.u_{0}\right)$, since $V_{k}(u) \geq V_{0}(u)$ on the entire line. It suffices to show that the minimum Dirichlet eigenvalue $E_{0} \geq-k^{2}$ for $V_{k}(u)$ with $k<0$, and $E_{0}>0$ when $k=0$. The function $V_{k}(u)$ for $k<0$ has global minimum value $-k^{2}$, which occurs at the unique point $u=\log (-2 k)$, and while for $k=0$ it is a strictly positive function. We use the Sturm comparison theorem for the potential $\tilde{V}_{A, u_{0}, E}(u):=A\left(u-u_{0}\right)^{2}-E$ for $A>$ 0 , so that $-\frac{d^{2}}{d u^{2}}+V_{A, u_{0}}(u)$ on $\left[u_{0}, \infty\right)$ is just a translated and scaled version of the harmonic oscillator potential on $[0, \infty)$, with eigenvalues shifted by $E$. For a given $\epsilon>0$, we take $E=k^{2}+\epsilon$ and then can choose $A>0$ small enough that

$$
\tilde{V}_{A, u_{0}, E}(u)=A\left(u-u_{0}\right)^{2}-k^{2}-\epsilon \leq V_{k}(u), \quad u_{0} \leq u<\infty .
$$

The Sturm comparison theorem in the singular case (see [17, Chapter 8 , Theorem 1]) now shows that the minimum eigenvalue $E_{0}$ of $V_{k}(u)$ has

$$
E_{0} \geq \tilde{E}_{0}-k^{2}-\epsilon,
$$

where $\tilde{E}_{0}$ is the minimum eigenvalue of $\tilde{V}_{A, 0,0}(u)=A u^{2}$ on $[0, \infty)$ with the same boundary conditions at $u=u_{0}$. It is well known that the oscillator Hamiltonian $-\frac{d^{2}}{d u^{2}}+u^{2}$ on the half-line $[0, \infty)$ with Dirichlet conditions at $u=0$ has a strictly positive spectrum with minimum eigenvalue $\tilde{\tilde{E}}_{0}=3$. Under rescaling of $A$ we still have $\tilde{E}_{0}>0$, and $E_{0}>-k^{2}$ follows from (2.9), taking $\epsilon$ small enough. For $k=0$ we may omit $\epsilon$, and so obtain $E_{0}>0$.

The Sturm theory implies monotonicity properties of the eigenvalues as the parameters $k, u_{0}$ are varied, with boundary conditions remaining fixed.

Theorem 2.2. Let $V_{k}(u)=\frac{1}{4} \mathrm{e}^{2 u}+k \mathrm{e}^{u}$. Let $E_{n, \alpha}\left(u_{0}, k\right)$ denote the nth eigenvalue $(n \geq 0)$ from the bottom of the spectrum of the operator $H=$ $-\frac{d^{2}}{d u^{2}}+V_{k}(u)$ on the half-line $\left[u_{0}, \infty\right)$ with boundary conditions

$$
(\cos \alpha) \psi\left(u_{0}\right)+(\sin \alpha) \psi^{\prime}\left(u_{0}\right)=0,
$$

with $\alpha$ being fixed. For each $n \geq 0$ the following hold:

(i) For fixed parameter $k \geq 0$, the $n$th eigenvalue $E_{n, \alpha}\left(u_{0}, k\right)$ is a strictly increasing function of $u_{0}$.

(ii) For fixed parameter $k<0$, the $n$th eigenvalue $E_{n, \alpha}\left(u_{0}, k\right)$ is a strictly increasing function of $u_{0}$, when $u_{0} \geq \log 2|k|$. 
The Schrödinger operator with Morse potential on the right half-line 335

(iii) For variable $k$, with fixed $u_{0}$, the $n$th eigenvalue $E_{n, \alpha}\left(u_{0}, k\right)$ is a strictly increasing function of $k$.

Proof. By a change of parameter $v=u-u_{0}$ we shift the left-hand endpoint to $v=0$. Then each of assertions (i)-(iii) above can be viewed as varying the potential, while keeping the left endpoint and the boundary condition fixed. In all cases the potentials are bounded below, and the variation increases the potential pointwise. The results then follow from comparison of the minimax characterization of the $n$th eigenvalue [56, XIII.1].

\section{Asymptotic formula for eigenvalue density}

We obtain estimates for the number of eigenvalues using a version of Weyl asymptotics, which says that the number of eigenvalues $E \leq T$ of a Hamiltonian $H=p^{2}+V(q)$ with $V(q)$ bounded below can be approximated by the volume in the phase space of the region $H \leq T$.

The half-line condition requires that we restrict the $q$-variable in the phase space to $\left[u_{0}, \infty\right)$ in estimating the phase space volume. The following result is a slight modification of a result of Titchmarsh [67, Theorem 7.4]) giving a rigorous estimate of this kind.

Theorem 3.1. Let $V(u)$ be a real-valued $C^{1}$-potential on $\left[u_{0}, \infty\right)$ which for all sufficiently large $u$ is increasing and convex downwards. Then the Schrödinger operator $-\frac{d^{2}}{d u^{2}}+V(u)$ on $\left[u_{0}, \infty\right)$ with boundary condition

$$
(\cos \alpha) \psi\left(u_{0}\right)+(\sin \alpha) \psi^{\prime}\left(u_{0}\right)=0
$$

has pure discrete simple spectrum. Let $N\left(T ; \alpha, u_{0}\right)$ count the number of eigenvalues $E_{n}<T$ for this boundary condition. Then there is a constant $T_{1}$ such that for all $T \geq T_{1}$ there is a unique solution $u_{T} \geq u_{0}$ to $V(u, T)=T$, and

$$
N\left(T ; \alpha, u_{0}\right)=\frac{1}{\pi} \int_{u_{0}}^{u_{T}} \sqrt{T-V(u)} d u+O(1)
$$

holds for $T_{1} \leq T<\infty$.

Proof. Suppose that $V(u)$ is increasing and convex downwards for $u \geq u_{1}$. The convexity condition implies for $u \geq u_{1}$ that $V(u)$ is strictly increasing and increases at least at a linear rate. Thus $V(u) \rightarrow \infty$ as $u \rightarrow \infty$, so the Schrödinger operator with given boundary conditions has a pure discrete simple spectrum. 
Titchmarsh [67, Theorem 7.4] proves estimate (3.1) assuming as hypotheses that $V(u)$ is continuous, increasing and convex downward on the whole interval $\left[u_{0}, \infty\right)$, i.e., that one can take $u_{1}=u_{0}$. A slight modification of his argument handles the case above. We set $T_{1}:=\max \left\{V(u): u_{0} \leq u \leq u_{1}\right\}$ For $T>T_{1}$, the strict increasing property of $V(u)$ for $u \geq u_{1}$ implies that the equation $V(u)=T$ has a unique solution, so that the set of values $\{u: V(u) \leq T\}$ is an interval $\left[u_{0}, u_{T}\right]$. Titchmarsh's argument estimates the number of oscillations in the real-valued $L^{2}$-solution $\psi(u ; E)$ with eigenvalue $E$ on $\left[u_{0}, \infty\right)$ (not necessarily satisfying the boundary conditions). We replace his integral estimate $0 \leq I_{2}(Y) \leq \frac{2}{3}$ with $I_{2}(Y)=O(1)$; the extra $C^{1}$-condition on $V(u)$ on the interval $\left[u_{0}, u_{1}\right]$ is imposed to guarantee that the relevant integrals exist.

We apply this result to the Morse potential. Note that the parameter $k$ in the Morse potential has no influence on the first two terms in the resulting asymptotic formula (3.2) below.

Theorem 3.2. For the Morse potential $V_{k}(u)=\frac{1}{4} \mathrm{e}^{2 u}+k \mathrm{e}^{u}$ on $\left[u_{0}, \infty\right)$ with boundary conditions $(\cos \alpha) \psi\left(u_{0}\right)+(\sin \alpha) \psi^{\prime}\left(u_{0}\right)=0$, the eigenvalue density satisfies

$$
N\left(T ; \alpha, u_{0}\right)=\frac{1}{\pi} \sqrt{T} \log \sqrt{T}+\frac{1}{\pi}\left(2 \log 2-1-u_{0}\right) \sqrt{T}+O(1)
$$

as $T \rightarrow \infty$, and the implied constant in the O-symbol depends on both $k$ and $u_{0}$.

Proof. The Morse potential $V_{k}(u)$ is increasing and convex downwards for all sufficiently large $u$, so Theorem 3.1 applies to give

$$
N(T, \alpha)=\frac{1}{\pi} \int_{u_{0}}^{u_{T}} \sqrt{T-V_{k}(u)} d u+O(1)
$$

as $T \rightarrow \infty$. For $k \geq 0$ the Morse potential is increasing and convex downward on the entire real line. However when $k<0$ there is a critical value $u_{1}$ to the left of which this no longer holds.

It remains to estimate the integral in (3.3), with $V_{k}(u)=\frac{1}{4} \mathrm{e}^{2 u}+k \mathrm{e}^{u}$. We first suppose that $u_{0}$ is such that $V_{k}(u)$ is increasing on $\left[u_{0}, \infty\right)$, which holds for all $u_{0}$ if $k \geq 0$ and for $u_{0} \geq u_{1}:=\log 2|k|$ if $k<0$. We assume in what follows that $u_{0} \geq \log (6|k|+1)$, and treat the case when $u_{0}<\log (6|k|+1)$ at the end of the proof. We suppose $T$ large enough to be in the region 
The Schrödinger operator with Morse potential on the right half-line 337

where $V_{k}(u)=T$ has a unique solution and introduce the positive constant $T^{*}$ given by $\left(T^{*}\right)^{2}=T+k^{2}$. We evaluate the integral

$$
I:=\int_{u_{0}}^{u_{T}} \sqrt{T-V_{k}(u)} d u=\int_{u_{0}}^{u_{T}} \sqrt{\left(T^{*}\right)^{2}-\left(\frac{1}{2} \mathrm{e}^{u}+k\right)^{2}} d u .
$$

We change variable $u$ to $w$ determined by

$$
w=\frac{T^{*}}{\frac{1}{2} \mathrm{e}^{u}+k} .
$$

Since $V_{k}(u)$ is monotone increasing on $\left[u_{0}, \infty\right)$ the change of variable is single valued, and on the integration interval $w$ decreases from $w_{0}=\frac{T^{*}}{\frac{1}{2} \mathrm{e}^{u_{0}}+k}$ to $w_{T}=1$. We have

$$
d w=-\frac{T^{*}}{\left(\frac{1}{2} \mathrm{e}^{u}+k\right)^{2}} \frac{1}{2} \mathrm{e}^{u} d u=-w\left(1-\frac{k w}{T^{*}}\right) d u,
$$

and obtain

$$
\begin{aligned}
I & =-\int_{w_{0}}^{1} \sqrt{\left(T^{*}\right)^{2}-\frac{\left(T^{*}\right)^{2}}{w^{2}}}\left(\frac{1}{1-\frac{k w}{T^{*}}}\right) \frac{d w}{w} \\
& =T^{*} \int_{1}^{w_{0}} \sqrt{1-\frac{1}{w^{2}}}\left(\frac{1}{1-\frac{k w}{T^{*}}}\right) \frac{d w}{w}
\end{aligned}
$$

in which $\frac{T^{*}}{w_{0}}=\frac{1}{2} \mathrm{e}^{u_{0}}+k$. We let $w=\mathrm{e}^{v}$, and obtain

$$
I=T^{*} \int_{0}^{v_{0}} \sqrt{1-\mathrm{e}^{-2 v}}\left(\frac{1}{1-\frac{k \mathrm{e}^{v}}{T^{*}}}\right) d v
$$

in which

$$
v_{0}=\log w_{0}=\log T^{*}-\log \left(\frac{1}{2} \mathrm{e}^{u_{0}}+k\right),
$$

where by hypothesis $\frac{1}{2} \mathrm{e}^{u_{0}}+k>|k|+\frac{1}{2}$. Now

$$
\mathrm{e}^{v}=w=\frac{T^{*}}{\frac{1}{2} \mathrm{e}^{u}+k}
$$

so

$$
T^{*}=\left(\frac{1}{2} \mathrm{e}^{u}+k\right) \mathrm{e}^{v}
$$


The hypothesis on $u_{0}>\log (6|k|+1)$ when $k<0$ ensures that

$$
0 \leq \frac{|k| \mathrm{e}^{v}}{T^{*}}<\frac{1}{2}
$$

Thus we may legally expand the integrand of (3.5) in a power series in $\frac{k \mathrm{e}^{v}}{T^{*}}$ and obtain $I=M+R$ with

$$
\begin{aligned}
M & :=T^{*}\left(\int_{0}^{v_{0}} \sqrt{1-\mathrm{e}^{-2 v}} d v\right) \\
R & :=T^{*}\left(\sum_{j=1}^{\infty}\left(\frac{k}{T^{*}}\right)^{j} \int_{0}^{v_{0}} \mathrm{e}^{j v} \sqrt{1-\mathrm{e}^{-2 v}} d v\right) .
\end{aligned}
$$

Now we have

$$
\begin{aligned}
M= & T^{*} v_{0}+T^{*}\left(\int_{0}^{v_{0}}\left(\sqrt{1-\mathrm{e}^{-2 v}}-1\right) d v\right) \\
= & T^{*}\left(\log T^{*}-\log \left(\frac{1}{2} \mathrm{e}^{u_{0}}+k\right)\right) \\
& +T^{*}\left(\int_{0}^{\infty}\left(\sqrt{1-\mathrm{e}^{-2 v}}-1\right) d v+O\left(\frac{1}{\left(T^{*}\right)^{2}}\right)\right)
\end{aligned}
$$

in which the constant in the $O$-symbol depends on $k$ and $u_{0}$. In the last line we used the estimate

$$
\left|\int_{v_{0}}^{\infty}\left(\sqrt{1-\mathrm{e}^{-2 v}}-1\right) d v\right| \leq \int_{\log T^{*}-c_{0}}^{\infty} \frac{1}{2} \mathrm{e}^{-2 v} d v \leq O\left(\frac{1}{\left(T^{*}\right)^{2}}\right)
$$

Now we use the identity

$$
\int_{0}^{\infty}\left(1-\sqrt{1-\mathrm{e}^{-2 v}}\right) d v=1-\log 2
$$

to obtain

$$
M=T^{*} \log T^{*}+\left(\log 2-1-\log \left(\frac{1}{2} \mathrm{e}^{u_{0}}+k\right)\right) T^{*}+O(1),
$$


The Schrödinger operator with Morse potential on the right half-line 339

and the constant in the $O$-symbol depends on $k$ and $u_{0}$. The remaining term $R=R_{1}+R_{2}$, in which

$$
\begin{aligned}
& R_{1}:=T^{*}\left(\sum_{j=1}^{\infty}\left(\frac{k}{T^{*}}\right)^{j} \int_{0}^{v_{0}} \mathrm{e}^{j v} d v\right), \\
& R_{2}:=T^{*}\left(\sum_{j=1}^{\infty}\left(\frac{k}{T^{*}}\right)^{j} \int_{0}^{v_{0}}\left(\sqrt{1-\mathrm{e}^{-2 v}}-1\right) \mathrm{e}^{j v} d v\right) .
\end{aligned}
$$

The first term above is

$$
\begin{aligned}
R_{1} & =T^{*}\left(\sum_{j=1}^{\infty}\left(\frac{k}{T^{*}}\right)^{j}\left(\frac{1}{j}\left(\mathrm{e}^{j v_{0}}-1\right)\right)\right) \\
& =T^{*}\left(-\log \left(1-\frac{k e^{v_{0}}}{T^{*}}\right)+\log \left(1-\frac{k}{T^{*}}\right)\right) \\
& =T^{*}\left(-\log \left(1-\frac{k}{\frac{1}{2} \mathrm{e}^{u_{0}}+k}\right)+O\left(\frac{1}{T^{*}}\right)\right) \\
& =T^{*}\left(\log \left(\frac{1}{2} \mathrm{e}^{u_{0}}+k\right)-\log \left(\frac{1}{2} \mathrm{e}^{u_{0}}\right)\right)+O(1) .
\end{aligned}
$$

The second term is estimated by

$$
\begin{aligned}
\left|R_{2}\right| & \leq T^{*} \sum_{j=1}^{\infty}\left(\frac{|k|}{T^{*}}\right)^{j} \int_{0}^{v_{0}} e^{(j-2) v} d v \\
& \leq|k|\left(1-e^{-v_{0}}\right)+\frac{|k|^{2}}{T^{*}} v_{0}+T^{*} \sum_{j=3}^{\infty}\left(\frac{|k|}{T^{*}}\right)^{j}\left(\frac{T^{*}}{\frac{1}{2} e^{u_{0}}+k}\right)^{j-2} \\
& \leq O(1)+\frac{k^{2}}{T^{*}} \sum_{j=3}^{\infty}\left(\frac{|k|}{\frac{1}{2} e^{u_{0}}+k}\right)^{j-2}=O(1)
\end{aligned}
$$

Putting these estimates together gives

$$
R=R_{1}+R_{2}=T^{*}\left(\log \left(\frac{1}{2} \mathrm{e}^{u_{0}}+k\right)-\log \left(\frac{1}{2} \mathrm{e}^{u_{0}}\right)\right)+O(1),
$$

and

$$
I=T^{*} \log T^{*}+\left(2 \log 2-1-u_{0}\right) T^{*}+O(1),
$$

provided that $u_{0} \geq \log (6|k|+1)$. 
In the remaining case where $u_{0} \leq \log (6|k|+1)$, we split the integral into a portion from $u_{0}$ to $u_{1}=\log (6|k|+1)$, and from $\left[u_{1}, \infty\right)$. The integral from $\left[u_{1}, \infty\right)$ is estimated by $(3.9)$ while that on $\left[u_{0}, u_{1}\right]$ by

$$
\int_{u_{0}}^{u_{1}} \sqrt{T-V_{k}(u)} d u=\int_{u_{0}}^{u_{1}} \sqrt{T-O(1)} d u=\left(u_{1}-u_{0}\right) \sqrt{T}+O\left(\frac{1}{\sqrt{T}}\right),
$$

in which the implied constant in the $O$-symbol depends on $u_{0}$ and $k$. Note that as $T^{*} \rightarrow \infty$, we have

$$
T^{*}=\sqrt{T+k^{2}}=\sqrt{T}\left(1+\frac{k^{2}}{2 T}+O\left(\frac{1}{T^{2}}\right)\right)=\sqrt{T}+O\left(\frac{1}{\sqrt{T}}\right) .
$$

Combining this with (3.10) shows that (3.9) remains valid for all intervals $\left[u_{0}, \infty\right)$. Next, substituting (3.11) in (3.9) yields

$$
I=\sqrt{T} \log \sqrt{T}+\left(2 \log 2-1-u_{0}\right) \sqrt{T}+O(1) .
$$

Substituting (3.12) in (3.3) gives the desired estimate (3.2) of $N(T, \alpha)$.

\section{Zeros of Whittaker functions in the $\mu$-variable}

For Dirichlet boundary conditions the eigenvalues of the Morse potential on the half-line are specified by Theorem 2.1 in terms of the zeros of Whittaker functions. We combine this with the results of Section 4 to deduce the distribution of zeros of these Whittaker functions in the $\mu$-variable. The function $Z_{1}(s)$ in the introduction is given as $Z_{1}(s)=Z\left(s+\frac{1}{2}\right)$, where $Z(\mu):=W_{\kappa, \mu}\left(\mathrm{e}^{u_{0}}\right)$ below.

Theorem 4.1. For fixed real parameters $\kappa$ and $u_{0}$ the Whittaker function

$$
Z(\mu):=W_{\kappa, \mu}\left(\mathrm{e}^{u_{0}}\right)
$$

is an entire function of $\mu$ of order 1 and maximal type. It is real valued on both the real axis and imaginary axis. It is an even function of $\mu$, i.e., it satisfies the "functional equation"

$$
Z(\mu)=Z(-\mu)
$$

(1) The zeros of $Z(\mu)$ lie on the real and imaginary axes. All zeros of $Z(\mu)$ are simple zeros, except for a possible zero at $\mu=0$, which if it occurs will be a double zero. 
The Schrödinger operator with Morse potential on the right half-line 341

(2) For each $\kappa$ the function $Z(\mu)$ has finitely many real zeros, which all lie in the interval $-|\kappa|<\mu<|\kappa|$. There are no real zeros when $\kappa \leq 0$.

(3) There are infinitely many imaginary zeros. The total number $N(Z(\mu))$ of zeros with $|\operatorname{Im}(\mu)| \leq T$ satisfies the asymptotic formula

$$
N(Z(\mu))=\frac{2}{\pi} T \log T+\frac{2}{\pi}\left(2 \log 2-1-u_{0}\right) T+O(1),
$$

as $T \rightarrow \infty$, where the $O$-constant depends on $\kappa$.

Proof. We set $x=\mathrm{e}^{u}$. It is well known that for fixed $\kappa$ and $x$ the Whittaker function $W_{\kappa, \mu}(x)$ is an entire function of $\mu$, see Appendix A. The function $W_{\kappa,-\mu}(x)$ satisfies Whittaker's differential equation with the same parameters $(\kappa, \mu)$, and it also has the same asymptotics as $x \rightarrow \infty$, namely

$$
W_{\kappa, \mu}(x)=\mathrm{e}^{-\frac{1}{2} x} x^{\kappa}\left(1+O\left(\frac{1}{x}\right)\right) .
$$

It follows that

$$
W_{\kappa,-\mu}(x)=W_{\kappa, \mu}(x)
$$

holds for all parameters $(\kappa, \mu)$ and positive real $x$. (It then holds universally under analytic continuation in the $x$-variable, see Appendix A.) The functional equation (4.2) follows, taking $x=\mathrm{e}^{u_{0}}$. This also implies that the function $G(\mu):=Z(\sqrt{\mu})$ is an entire function of $\mu$.

We next establish the real symmetry when $\kappa$ is real and $x=e^{u_{0}}$ is positive-real valued (on the principal branch of the Whittaker function). For $2 \mu$ not an integer we have the representation [74, Section 16.41]

$$
W_{\kappa, \mu}(x)=\frac{\Gamma(-2 \mu)}{\Gamma\left(\frac{1}{2}-\kappa-\mu\right)} M_{\kappa, \mu}(x)+\frac{\Gamma(2 \mu)}{\Gamma\left(\frac{1}{2}-\kappa+\mu\right)} M_{\kappa,-\mu}(x) .
$$

Now we assert that, for real $\kappa$ and positive real $x$, applying complex conjugation to (5.4) yields

$$
W_{\kappa, \bar{\mu}}(x)=\overline{W_{\kappa, \mu}(x)} .
$$

To justify (4.6), we note that for positive real $x$, on the principal branch of the Whittaker $M$-function we have

$$
M_{\bar{\kappa}, \bar{\mu}}(x)=\overline{M_{\kappa, \mu}(x)}
$$


(see (A.5) in the appendix) and that $\Gamma(\bar{s})=\overline{\Gamma(s)}$ for $s \in \mathbb{C}$, since it is real on the real axis. This justifies (4.6), and in fact for real $\kappa$ one has $\overline{W_{\kappa, \mu}(z)}=$ $W_{\kappa, \bar{\mu}}(\bar{z})$ for complex $z$ with $|\arg (z)|<\pi$. We conclude using (5.4) that for real $\kappa$ and positive real $x$, the function $W_{\kappa, \mu}(x)$ is real when $\mu$ is on the real axis. In addition, under the same assumptions, the conjugation symmetry (5.4) together with symmetry (4.4) implies that $W_{\kappa, \mu}(x)$ is real when $\mu=\mathrm{it}$ is on the imaginary axis.

For real $\kappa$ and positive real $x$, a growth bound deduced from the contour integral representation given in formula (A.9) of Appendix A implies that the function $Z(\mu)=W_{\kappa, \mu}\left(\mathrm{e}^{u_{0}}\right)$ is an entire function of order at most 1 . The fact that $Z(\mu)$ is entire of order 1 and maximal type will follow from the zero counting formula (4.3), asserting that for some $C>0$ it has at least $C T \log T$ zeros in the disk $|z|<T$ as $T \rightarrow \infty$. For any entire function of order less than 1 or of order 1 and finite type necessarily has $O(T)$ zeros in each disk $|z| \leq T$ as $T \rightarrow \infty$ ([66, Section 8.75]). We now establish (1)-(3).

(1) Apply Theorem 2.1 for the Morse potential $V_{k}(u)$ with $k=-\kappa$. Then the zeros of $Z(\mu):=W_{\kappa, \mu}\left(\mathrm{e}^{u_{0}}\right)$ correspond to the $L^{2}$-eigenfunction $\psi\left(u_{0},-\mu^{2}\right)=0$, corresponding to $E=-\mu^{2}$ being a Dirichlet boundary condition eigenvalue of the Morse potential Schrödinger equation on the half-line $\left[u_{0}, \infty\right)$. This boundary value problem is self-adjoint, so $E$ is real. Thus $\mu$ must be real or pure imaginary.

The Schrödinger operator spectrum is simple, therefore each value of $E$ occurs once. Both roots in $\mu$ of $E=-\mu^{2}$ produce a zero of the Whittaker function, due to the symmetry $W_{\kappa, \mu}(x)=W_{\kappa,-\mu}(x)$. These zeros must therefore be simple, except for $\mu=0$, where a double zero must occur since $W_{\kappa,-\mu}(x)$ is an even function of $\mu$.

(2) Theorem 2.1 establishes that the Schrödinger spectrum for Dirichlet boundary conditions is pure discrete and is bounded below by $E=-\mu^{2}>$ $-k^{2}$, and by $E>0$ when $k \geq 0$. Thus there can only be finitely many roots $\mu$ on the real axis, all with $0 \leq \mu^{2}<k^{2}$ so that $-|\kappa|<\mu<|\kappa|$, and there are no real roots when $k \geq 0$, i.e. $\kappa \leq 0$.

(3) The asymptotic estimate (4.3) follows from Theorem 3.2. A pair of zeros $\mu= \pm \mathrm{i} t$ corresponds to a single Dirichlet eigenvalue $E=-\mu^{2}=t^{2}$ of the Schrödinger operator, and the condition $|\mu| \leq T$ corresponds to the bound $E<T^{2}$. Thus we obtain from the eigenvalue asymptotic (3.2) that

$$
N(Z(\mu))=N\left(T ; 0, u_{0}\right)=\frac{2}{\pi} T \log T+\frac{2}{\pi}\left(2 \log 2-1-u_{0}\right) T+O(1),
$$

as required. 
Remark. For the case $k=0$, and $\Re(w)>0$ the Whittaker function is related to the $K$-Bessel function (MacDonald function) by

$$
K_{\mu}(w)=\sqrt{\frac{\pi}{2 w}} W_{0, \mu}(2 w) .
$$

The $K$-Bessel function for positive real $w=\mathrm{e}^{u}$ is known to have all its zeros on the imaginary axis. According to Erdélyi et al. [28, Vol II, 7.13 .2 (19)] an asymptotic formula is available when $\mu=\mathrm{it}$ is on the imaginary axis with $w=x=\mathrm{e}^{u}$. This formula states (taking $M=1$ ) that, for $t>x>0$,

$$
\begin{aligned}
& K_{\mathrm{it}}(x)=\sqrt{2}\left(t^{2}-x^{2}\right)^{-1 / 4} \mathrm{e}^{-\frac{1}{2} \pi t}\left(\sqrt{\pi} \sin \left(t \cosh ^{-1}\left(\frac{t}{x}\right)-\sqrt{t^{2}-x^{2}}+\frac{\pi}{4}\right)\right. \\
& \left.(4.8)+O\left(\frac{1}{\sqrt{t^{2}-x^{2}}}\right)\right) .
\end{aligned}
$$

These asymptotics agree with that of Theorem 4.1.

\section{Eigenvalue interpretations for Riemann zeta zeros}

There is considerable circumstantial evidence for the existence of a spectral interpretation of the Riemann zeta zeros, see $[4,38]$. Indeed there are several known operator-theoretic interpretations of zeta zeros. There is a scattering theory interpretation of the Riemann zeta zeros arising from work of Pavlov and Faddeev [52] concerning the Laplacian acting on the modular surface. A slightly different version of this interpretation is given by Lax and Phillips [44], see in particular [44, Appendix 2 to Section 7]. Recently, an operator formulation of the Lax-Phillips approach was given by Uetake [72]. There are also interpretations of the zeta zeros in terms of eigenvalues of operators on various function spaces, given by Connes [18]. The formulation of Connes permits the construction of such an operator on a Hilbert space that has eigenvalues that detects only those zeta zeros that are on the critical line, up to a fixed multiplicity. This approach was extended to automorphic $L$-functions by Soulé [65] and Deitmar [20]. Meyer [47,48] gave an unconditional formulation of an operator on a more general Banach space whose eigenvalues detect all zeta zeros, including those that are off the critical line if the Riemann hypothesis fails. There is also recent work of Sierra [59-62] and Sierra and Townsend [63] concerning quantum-mechanical models for the zeta zeros, and possible "Hilbert-Polya" operators. Burnol [15] has constructed a specific "toy model" operator of the integral-differential form considered by Sierra, based on his earlier work [13]. 
We recently observed $[41,42]$ that there is a natural candidate for a "Hilbert-Polya" operator, using the framework of the de Branges Hilbert spaces of entire functions, provided that the Riemann hypothesis holds. This interpretation leads to a possible connection with Schrödinger operators on a half-line, described below.

We first review some aspects of the de Branges theory. The de Branges theory of Hilbert spaces of entire functions [7] assigns to an entire function $E(z)$ of a special type called a structure function a reproducing kernel Hilbert space of entire functions $\mathcal{H}(E(z))$, together with a (generally unbounded) linear operator $M_{z}: f(z) \mapsto z f(z)$ defined on the domain $\mathcal{D}_{z}:=$ $\{f(z) \in \mathcal{H}(E(z)): z f(z) \in \mathcal{H}(E(z))\}$. This operator is closed and symmetric, with deficiency indices $(1,1)$, so that it has a one-parameter family of self-adjoint extensions, parametrized by the unit circle $S^{1}=U(1)$. A structure function $E(z)$ is any entire function satisfying

$$
|E(z)|>|E(\bar{z})|, \quad \text { when } \quad \operatorname{Im}(z)>0
$$

Functions $E(z)$ with this property are sometimes called Hermite-Biehler functions (see [46, Chapter VII]). Any entire function can be uniquely represented as $E(z)=A(z)-\mathrm{i} B(z)$, in which $A(z), B(z)$ are entire functions that are real on the real axis. If we let $E^{\sharp}(z):=\overline{E(\bar{z})}$, then

$$
A(z)=\frac{1}{2}\left(E(z)+E^{\sharp}(z)\right), \quad B(z)=\frac{1}{2 \mathrm{i}}\left(E(z)-E^{\sharp}(z)\right) .
$$

The Hermite-Biehler property (5.1) has the consequence that the associated $A(z)$ and $B(z)$ have only real zeros, and these zeros interlace (allowing multiple zeros, counting multiplicity) (cf. [40, Lemma 2.2]). The Hilbert space $\mathcal{H}(E(z))$ is always nonempty, and it consists of all entire functions satisfying certain growth restrictions (compared to $E(z), E^{\sharp}(z)$ ) in the upper half-plane. One particular self-adjoint extension of $\left(M_{z}, \mathcal{D}_{z}\right)$ has pure discrete simple spectrum located at the zeros of $A(z)$, and another has pure discrete simple spectrum located at the zeros of $B(z)$.

The de Branges theory also gives a Fourier-like transform, depending on the structure function, that gives an isometry to a new Hilbert space on which the multiplication operator becomes a $2 \times 2$ matrix system of linear differential operators, called a canonical system; we call this the de Branges transform. Here a canonical system is a family of differential equations, depending on $z \in \mathbb{C}$ as a parameter, on an interval $[a, b]$ in which we 
allow $0 \leq a<b \leq \infty$, given by

$$
\frac{d}{d t}\left[\begin{array}{l}
A(t, z) \\
B(t, z)
\end{array}\right]=z \mathbf{J M}(t)\left[\begin{array}{l}
A(t, z) \\
B(t, z)
\end{array}\right]
$$

in which

$$
\mathbf{J}=\left[\begin{array}{cc}
0 & -1 \\
1 & 0
\end{array}\right], \quad \mathbf{M}(t)=\left[\begin{array}{ll}
\alpha(t) & \beta(t) \\
\beta(t) & \gamma(t)
\end{array}\right]
$$

with $\mathbf{M}(t)$ being a positive semidefinite real matrix-valued function on the interval $(a, b)$, with coefficients being measurable real valued functions of $t$. In fact, de Branges's formulation of his theory [7] formulates results using an integral equation which is an integrated form of the canonical system, in order to deal with smoothness issues; he does not use canonical systems. However the use of a canonical system makes comparison with Schrödinger operator formulation easier, cf. [57]. In general, the de Branges transform to the canonical system is not known explicitly, but it has been determined in a number of "exactly solvable" examples, see [6], [7, Chapter 3].

A special subclass of canonical systems can be nonlinearly transformed to a pair of Schrödinger operators on an interval or a half-line, a transformation that squares the eigenvalues. Such canonical systems are a subclass of those whose canonical matrix $\mathbf{M}(t)$ is diagonal and invertible almost everywhere. de Branges [7, Section 4] proves a result stating that a structure function has an associated canonical system that is diagonal whenever $A(z)$ is an even function and $B(z)$ is an odd function. For such diagonal canonical systems, one may monotonically rescale the time variable so that the coefficient matrix becomes

$$
\mathbf{M}(t)=\left[\begin{array}{cc}
\alpha(t) & 0 \\
0 & \gamma(t)
\end{array}\right]
$$

with determinant 1 almost everywhere, on a rescaled interval $[\tilde{a}, \tilde{b}]$ with $-\infty<\tilde{a}<\tilde{b} \leq \infty$. If these coefficients are smooth enough, then the transform gives a (supersymmetric) pair of transformed Schrödinger operators $-\frac{d^{2}}{d t^{2}}+V^{ \pm}(t)$ having potentials

$$
V^{ \pm}(t):=W(t)^{2} \pm W^{\prime}(t)=\frac{1}{4}\left(\frac{\alpha^{\prime}(t)}{\alpha(t)}\right)^{2} \pm \frac{1}{2}\left(\frac{\alpha^{\prime \prime}(t) \alpha(t)-\alpha^{\prime}(t)^{2}}{\alpha(t)^{2}}\right)
$$


with associated superpotential

$$
W(t):=\frac{1}{2} \frac{\alpha^{\prime}(t)}{\alpha(t)},
$$

where $\alpha^{\prime}(t)=\frac{d}{d t} \alpha(t)$. These two Schrödinger operators have related boundary conditions at the left endpoint, such that they are self-adjoint with identical discrete spectrum, with the exception of the eigenvalue 0. One of them has Dirichlet boundary conditions, the other has boundary conditions that depend on the eigenvalue. There are a number of known examples where, starting from the structure function, all these steps can be carried out explicitly to obtain such a pair of Schrödinger operators. This is the case for the Morse potential for certain parameter values, using the fact that it is a shape-invariant potential treatable by supersymmetry, cf. [19, Section 4.1], see $[43,51]$.

In [40-42] we considered de Branges spaces associated to the Riemann zeta function and more general $L$-functions. In particular, we associate to the Riemann $\xi$-function a family of entire functions depending on the real parameter $h$, given for $h \neq 0$ by

$$
\begin{aligned}
E_{h}(z):= & \frac{1}{2}\left(\xi\left(\frac{1}{2}+h-\mathrm{i} z\right)+\xi\left(\frac{1}{2}-h-\mathrm{i} z\right)\right) \\
& -\frac{i}{2 h}\left(\xi\left(\frac{1}{2}+h-\mathrm{i} z\right)-\xi\left(\frac{1}{2}-h-\mathrm{i} z\right)\right),
\end{aligned}
$$

and for $h=0$ by

$$
E_{0}(z):=\xi\left(\frac{1}{2}-\mathrm{i} z\right)+\xi^{\prime}\left(\frac{1}{2}-\mathrm{i} z\right),
$$

with $\xi^{\prime}(s)=\frac{d}{d s} \xi(s)$. We show in [40, Theorem 2.1] that for $|h| \geq \frac{1}{2}$ each member of this family is unconditionally a de Branges structure function, and, conditionally on the Riemann hypothesis, that they are structure functions for all nonzero real $h$. Furthermore, we show in [41, Theorem 1] that the Riemann hypothesis holds if and only if the entire function $E_{0}(z)$ in (5.5) is a de Branges structure function. Assuming $\mathrm{RH}$, a theorem of de Branges [7, Section 47] applies to assert that the canonical system associated to $E_{0}(z)$ by the de Branges transform has matrix function $\mathbf{M}(t)$ of diagonal form.

To summarize, if the Riemann hypothesis holds, the de Branges theory predicts the existence of a "Hilbert-Polya" operator given as a diagonal canonical system, specifically associated to the structure function (5.5). Now 
we can ask if one can nonlinearly transform this diagonal canonical system to a pair of Schrödinger operators. As obstacles, we do not know whether the resulting diagonal matrix function $\mathbf{M}(t)$ will be almost everywhere invertible, and even if it is, we do not know whether the resulting matrix coefficients (5.3) will be smooth enough to permit the nonlinear transform (5.4). Here we speculate that the smoothness conditions will be the main obstacle, and that one may wish to consider this transform in the sense of generalized functions. (This is a suitable topic for further research in its own right.) In any case, if this transform were possible, there would then exist a "natural" Schrödinger operator on a half-line, having a (possibly distributional) "Hilbert-Polya" potential that, for Dirichlet boundary conditions, has as simple eigenvalues $\gamma^{2}$ corresponding to $\rho=\frac{1}{2} \pm i \gamma$ being a pair of zeta zeros. One can then attempt to reverse-engineer this potential, or in any case, to reverse-engineer the matrix coefficient functions of the associated canonical system.

For the Morse potential treated here the "toy model" aspect can be extended further, to the level of de Branges spaces and canonical systems. That is, there is a family of de Branges spaces associated to the Morse potentials, described in [43], where the nonlinear transformation to a Schrödinger operator on a half-line can be justified. As an example, one can show that for real $u$ the function

$$
E_{0}(u, z):=\mathrm{e}^{\frac{1}{2}\left(\mathrm{e}^{u}-u\right)} W_{\frac{1}{2}, \mathrm{i} z}\left(\mathrm{e}^{u}\right)-\mathrm{i} z e^{-\frac{1}{2}\left(\mathrm{e}^{u}+u\right)} W_{-\frac{1}{2}, \mathrm{i} z}\left(\mathrm{e}^{u}\right)
$$

is a de Branges structure function, and the transforms above convert it to a Schrödinger operator with Morse potential with parameter $k=-\frac{1}{2}$ on a corresponding half-line $[u, \infty)$. In particular, the self-adjoint extension of the de Branges multiplication operator associated to

$$
A(z)=\mathrm{e}^{-\frac{1}{2}\left(\mathrm{e}^{u}-u\right)} W_{\frac{1}{2}, \mathrm{i} z}\left(\mathrm{e}^{u}\right)
$$

is transformed to this Schrödinger operator with Dirichlet boundary conditions at the left endpoint. This example shows that there is no apparent obstacle at the level of asymptotic distribution of eigenvalues to finding a diagonal canonical system transformable to an associated Schrödinger potential that encodes the zeta zeros. Such an (integrated) potential must increase exponentially as $t \rightarrow \infty$ to have the correct asymptotics of zero densities.

We next address the feature that these "toy models" reproduce only the main term asymptotics of the distribution of zeta zeros, but not the fine structure of these zeros. Namely, the Riemann zeta zeros are believed to 
have local density statistics with fluctuations described by the GUE distribution of random matrix theory, see [38]. These GUE fluctuations encode properties of the distribution of primes, and they partially manifest themselves in the fact that the error term $O(\log T)$ for zeta zero asymptotics in (1.4) is nontrivial. In contrast the zeros of the Whittaker functions have normalized local density statistics which provably have no fluctuations, i.e., the normalized zeros will be completely regularly spaced, a fact that corresponds to the $O(1)$ error term in (1.3). This fact is not so far removed from the zeta function itself, because in [41] a similar phenomenon was observed for zeros of differenced Dirichlet $L$-functions, whenever one moves off the critical line to a line $\operatorname{Re}(s)=\frac{1}{2}+\delta$, for some $\delta>0$. The zeros of these differenced $L$-functions correspond to vanishing of the real part of the $L$-function $L\left(\frac{1}{2}+\delta+\mathrm{i} t, \chi\right)$. The asymptotics of these differenced $L$-function zeros is known to have the same main term as the zeta zero asymptotics, but also to have a $O(1)$ remainder term, just as in these "toy models." Furthermore Lagarias [41] showed that these differenced $L$-function zeros do have an eigenvalue interpretation for a multiplication operator in a suitable de Branges space, assuming RH. This fact shows that the GUE phenomenon for the zeta zeros is (in some sense) confined to the critical line $\operatorname{Re}(s)=\frac{1}{2}$. We take this to mean that, assuming RH holds, the de Branges space with structure function (5.5) will have some unusual features of its associated canonical system. Perhaps these will be badly behaved coefficients, so that if there is an associated "Hilbert-Polya" Schrödinger operator, its potential will be very badly behaved, and cannot be treated as a function.

In any case a desire to explain GUE suggests further consideration of the question: what conditions must a Schrödinger potential $V(x)$ satisfy in order to have eigenvalues that reproduce the Weyl asymptotics of the zeta zeros and also obey local normalized GUE statistics?

\section{Concluding remarks}

From the perspective of de Branges spaces, the natural operator to consider is a canonical system. Indeed, if the Riemann hypothesis holds, a canonical system "Hilbert-Polya" operator must exist with structure function (5.5) whose (real) eigenvalues are the imaginary parts of the zeta zeros. This paper is motivated by the observation that under some conditions a canonical system can be (nonlinearly) transformed to the familiar form of a one-dimensional Schrödinger operator on a half-line, also known as a singular Sturm-Liouville problem. This raises the possibility that the "Hilbert-Polya" operator proposed for zeta zeros might be realizable by 
such a Schrödinger operator, which falls squarely in the framework of quantum mechanics.

The existence of this nonlinear transformation reflects the fact that the de Branges theory generalizes part of Weyl's theory of singular SturmLiouvlille problems, cf. [29]. The Morse potential example considered here is a singular Sturm-Liouville problem which falls within Weyl's theory. That theory assigns to each such problem an analytic function, the principal Weyl-Titchmarsh $m$-function, which encodes information about the spectral data of the problem, encoded in the initial conditions. This function is discussed in Appendix B. This function is analytic in the upper halfplane and has positive imaginary part there, i.e., it is a so-called Herglotz function.

One may also associate directly to a de Branges structure function $E(z)$ the function

$$
m_{E}(z):=-\frac{B(z)}{A(z)}
$$

which we will here call the de Branges m-function. It is a Herglotz function associated to the de Branges multiplication operator $\left(M_{z}, \mathcal{D}_{z}\right)$ which plays a role analogous to that of the principal Weyl-Titchmarsh $m$-function for Schrödinger operators. That is, it encodes certain information on the spectrum of self-adjoint extensions of this symmetric operator. More precisely, under the de Branges transform it equals a "classical" $m$-function attached to a Dirac operator, in the sense of Levitan and Sargsan [45, Chapter 3]. This Dirac operator is directly constructed from the canonical system (5.2), by a recipe we omit here. In the case of the de Branges structure function (5.6) above, using a Whittaker function identity [1, (13.4.30)], we obtain

$$
-\frac{B(z)}{A(z)}=-\mathrm{e}^{-\mathrm{e}^{u}} \frac{z W_{-\frac{1}{2}, \mathrm{i} z}\left(\mathrm{e}^{u}\right)}{W_{\frac{1}{2}, \mathrm{i} z}\left(\mathrm{e}^{u}\right)}=-\frac{1}{z}\left[\mathrm{e}^{-\mathrm{e}^{u}}\left(-\frac{W_{\frac{3}{2}, \mathrm{i} z}\left(\mathrm{e}^{u}\right)}{W_{\frac{1}{2}, \mathrm{i} z}\left(\mathrm{e}^{u}\right)}+\mathrm{e}^{u}-1\right)\right]
$$

For comparison, in Appendix B we determine the principal Weyl-Titchmarsh $m$-function for the Morse potential on the right half-line (Theorem B.1). Formula (6.2) should be compared with Theorem B.1 with parameter $k=-\frac{1}{2}$. The computations in Appendix B may be useful as a guide for determining relations between Weyl-Titchmarsh $m$-functions and suitable de Branges $m$-functions. 


\section{Appendix A: Whittaker functions in the complex domain}

We recall here facts about Whittaker functions $W_{\kappa, \mu}(z)$ as functions of three complex variables $(\kappa, \mu, z)$. They are a type of confluent hypergeometric function, and are single valued in the variables $\kappa$ and $\mu$, and multi-valued in the $z$ variable, with a singular point at $z=0$.

The standard confluent hypergeometric function ${ }_{1} F_{1}(\alpha, \beta ; z)$ is given by the power series expansion

$$
{ }_{1} F_{1}(\alpha, \beta ; z):=\frac{\Gamma(\beta)}{\Gamma(\alpha)} \sum_{j=0}^{\infty} \frac{\Gamma(\alpha+j)}{\Gamma(\beta+j)} \frac{z^{j}}{j !} .
$$

The normalized function

$$
{ }_{1} \tilde{F}_{1}(\alpha, \beta ; z):=\frac{1}{\Gamma(\beta)}{ }_{1} F_{1}(\alpha, \beta ; z)
$$

is an entire function of three complex variables $(\alpha, \beta, z) \in \mathbb{C}^{3}$, as can be read off from the uniform convergence properties of expansion (A.1) on compact subsets of $\mathbb{C}^{3}$, observing that $\frac{1}{\Gamma(\beta+j)}$ is entire and $\frac{\Gamma(\alpha+j)}{\Gamma(\alpha)}$ is a polynomial in $\alpha$, cf. [8, Section 1.3]. (The unnormalized function ${ }_{1} F_{1}(\alpha, \beta ; z)$ has simple poles at $\beta=0,-1,-2, \ldots$ for most $(\alpha, z)$.) It satisfies the confluent hypergeometric differential equation

$$
z \frac{d^{2} F}{d z^{2}}+(\beta-z) \frac{d F}{d z}-\alpha F=0,
$$

which has a regular singular point at $z=0$ and an irregular singular point at $z=\infty$, of irregularity index one ([64]).

The Whittaker functions $M_{\kappa, \mu}(z)$ and $W_{\kappa, \mu}(z)$ were given by Whittaker [73] in 1904 as particular solutions to the differential equation

$$
\frac{d^{2} F}{d z^{2}}+\left(-\frac{1}{4}+\frac{\kappa}{z}+\frac{\frac{1}{4}-\mu^{2}}{z^{2}}\right) F=0 .
$$

This equation also has a regular singular point at $z=0$ and an irregular singular point at $z=\infty$ on the Riemann sphere, of irregularity index one. Whittaker sets

$$
M_{\kappa, \mu}(z):=\mathrm{e}^{-\frac{z}{2}} z^{\frac{1}{2}+\mu}{ }_{1} F_{1}\left(\frac{1}{2}-\kappa+\mu, 2 \mu+1 ; z\right),
$$

with $z^{-\mu}=\mathrm{e}^{\mu \log z}$, and the $z$-plane is cut along the negative real axis. This defines the principal branch of the Whittaker $M$-function. Under analytic 
continuation in the $z$-variable, this function is multivalued, with multivaluedness arising entirely from the function $z^{-\mu}$. It also has poles at $\mu=$ $0,-\frac{1}{2},-1, \ldots$ and to eliminate these (excluding $\mu=0$ ) Buchholz $[8$, p. 12] introduces the normalized function $\mathcal{M}_{\kappa, \mu}(z)$, defined by

$$
\mathcal{M}_{\kappa, \mu}(z):=\frac{1}{\Gamma(1+2 \mu)} M_{\kappa, \mu}(z)
$$

The Whittaker function $W_{\kappa, \mu}(z)$ is specified by the asymptotic property of having rapid decrease as $z=x \rightarrow \infty$ along the positive real axis. Whittaker [73] defined it using two integral representations given in [74, Section 16.12]. In terms of the confluent hypergeometric function above we have $[70$, p. 170]

$$
\begin{aligned}
W_{\kappa, \mu}(z)= & \frac{\Gamma(-2 \mu) z^{\frac{1}{2}+\mu} \mathrm{e}^{-\frac{z}{2}}}{\Gamma\left(\frac{1}{2}-\kappa-\mu\right)}{ }_{1} F_{1}\left(\frac{1}{2}-\kappa+\mu, 2 \mu+1 ; z\right) \\
& +\frac{\Gamma(2 \mu) z^{\frac{1}{2}-\mu} \mathrm{e}^{-\frac{z}{2}}}{\Gamma\left(\frac{1}{2}-\kappa+\mu\right)}{ }_{1} F_{1}\left(\frac{1}{2}-\kappa-\mu,-2 \mu+1 ; z\right) .
\end{aligned}
$$

This formula can alternatively be written as

$$
W_{\kappa, \mu}(z)=\frac{\Gamma(-2 \mu)}{\Gamma\left(\frac{1}{2}-\kappa-\mu\right)} M_{\kappa, \mu}(z)+\frac{\Gamma(2 \mu)}{\Gamma\left(\frac{1}{2}-\kappa+\mu\right)} M_{\kappa,-\mu}(z),
$$

which incidentally shows that $W_{\kappa, \mu}(z)=W_{\kappa,-\mu}(z)$. Formula (A.7) directly defines $W_{\kappa, \mu}(z)$ as an analytic function of three complex variables, in the region $(\kappa, \mu) \in \mathbb{C}^{2}$ and all $z$ in the complex plane cut along the negative real axis. (The analyticity is read off using function (A.2).) We term $W_{\kappa, \mu}(z)$ on this region the principal branch of the Whittaker function. Under analytic continuation of this function in the $z$-variable, this function is multivalued, and becomes single valued on a logarithmic covering surface around the point $z=0$, remaining an entire function of $\kappa$ and $\mu$ on every branch above a fixed $z$. (For certain values of $\kappa$ and $\mu$ it may be single valued on a finite cover of $\mathbb{C}^{*}=\mathbb{C} \backslash\{0\}$.)

The function $W_{\kappa, \mu}(z)$ also has the contour integral representation [74, Section 16.12]

$$
\begin{aligned}
W_{\kappa, \mu}(z)= & \Gamma\left(\frac{1}{2}+\kappa-\mu\right) \mathrm{e}^{-\frac{z}{2}} z^{\kappa} \\
& \times\left(-\frac{1}{2 \pi \mathrm{i}} \int_{C}(-t)^{-\kappa-\frac{1}{2}+\mu}\left(1+\frac{t}{z}\right)^{\kappa-\frac{1}{2}+\mu} \mathrm{e}^{-t} d t\right),
\end{aligned}
$$


in which the contour $C$ goes from $+\infty$ to $0^{+}$and back by a "keyhole" contour around the positive real axis, keeping $t=-z$ outside the contour, with $\arg (z)$ taking the principal value, $|\arg (-t)| \leq \pi$, and $\arg \left(1+\frac{t}{z}\right)$ taking that value which goes to zero at $t \rightarrow 0$ by a path outside the contour. If we restrict $z$ to the positive real axis, one obtains by estimating the contour integral (A.9) that

$$
W_{\kappa, \mu}(z)=O\left(\mathrm{e}^{C|\mu| \log (|\mu|+2)}\right)
$$

viewed as a function of $\mu$, for fixed $\kappa$ and $z$, a constant $C$ depending on $\kappa$ and $z$. This implies that $W_{\kappa, \mu}(z)$ is an entire function of order at most 1 in the $\mu$-variable.

The distinguishing property of the principal branch of $W_{\kappa, \mu}(z)$ is its rapid decrease along the real axis $z=x$ as $x \rightarrow \infty$. All other linearly independent solutions to Whittaker's equation (A.4) increase rapidly (in absolute value) along the positive real axis as $x \rightarrow \infty$. The rapid decrease property of the principal branch of the Whittaker function holds uniformly as $|z| \rightarrow \infty$ on the angular sector $|\arg (z)|<\frac{\pi}{2}-\epsilon$ for any fixed $\epsilon>0$. Furthermore, for fixed $(\kappa, \mu)$, it has on the larger angular sector $-\pi+\epsilon<\arg (z)<\pi-\epsilon$, an asymptotic expansion [74, Section 16.3] which gives

$$
W_{\kappa, \mu}(z)=\mathrm{e}^{-\frac{z}{2}} z^{\kappa}\left(1+O\left(\frac{\mu^{2}-\left(\kappa-\frac{1}{2}\right)^{2}}{z}\right)\right) .
$$

The Whittaker function solutions having this rapid decrease property glue together smoothly in the $(\kappa, \mu)$ variables (uniformly with $|\kappa|^{2}+|\mu|^{2}<R$ for any fixed $R$ ) to form entire functions of $(\kappa, \mu) \in \mathbb{C}^{2}$, yielding the principal branch defined above. For certain values of $\kappa$ and $\mu$ we have a finite covering of $\mathbb{C}^{*}$; in these cases there are a finite number of other branches.

\section{Appendix B: Weyl-Titchmarsh $m$-functions for Morse potentials}

For Schrödinger operators on the half-line $\left[u_{0}, \infty\right)$ with a real-valued potential, the Weyl-Titchmarsh $m$-function $m\left(u_{0}, E\right)$ is an analytic function defined on $E \in \mathbb{C} \backslash \mathbb{R}$, which encodes information about the spectrum of the Schrödinger operator for various constant boundary conditions. It maps the upper half-plane $\Im(E)>0$ into itself, and its values on the lower half-plane $\Im(E)<0$ satisfy $m\left(u_{0}, \bar{E}\right)=\overline{m\left(u_{0}, E\right)}$. Spectral information is extractable from its asymptotics in the upper half-plane as the real axis is approached, 
see [32]. In general it is not defined on the real axis, but in the case where the spectrum is pure discrete then it has a meromorphic extension to the whole plane $\mathbb{C}$, which is real valued on the real axis.

To define it, let $\theta_{\alpha}(u ; E), \varphi_{\alpha}(u, E)$ denote the solutions to the initial value problems

$$
\left(-\frac{d^{2}}{d u^{2}}+V(u)\right) \psi(u)=E \psi(u)
$$

with

$$
\left[\begin{array}{c}
\theta_{\alpha}\left(u_{0}, E\right) \\
\theta_{\alpha}^{\prime}\left(u_{0}, E\right)
\end{array}\right]=\left[\begin{array}{c}
\cos \alpha \\
\sin \alpha
\end{array}\right] \text { and } \quad\left[\begin{array}{c}
\varphi_{\alpha}\left(u_{0}, E\right) \\
\varphi_{\alpha}^{\prime}\left(u_{0}, E\right)
\end{array}\right]=\left[\begin{array}{c}
-\sin \alpha \\
\cos \alpha
\end{array}\right]
$$

We define the $m$-function $m_{\alpha}(E):=m_{\alpha}\left(u_{0}, E\right)$ by the condition that

$$
\psi\left(u_{0}, E\right):=\theta_{\alpha}\left(u_{0}, E\right)+m_{\alpha}(E) \varphi_{\alpha}\left(u_{0}, E\right)
$$

belongs to $L^{2}\left(\left[u_{0}, \infty\right) ; d u\right)$. The case $\alpha=0$ gives the principal WeylTitchmarsh $m$-function, where $\varphi_{0}$ satisfies Dirichlet boundary conditions at the left endpoint, and $\theta_{0}$ satisfies Neumann boundary conditions; we write $m\left(u_{0}, E\right):=m_{0}\left(u_{0}, E\right)$. For the principal $m$-function we have the alternate formula

$$
m\left(u_{0}, E\right)=\frac{\psi^{\prime}\left(u_{0}, E\right)}{\psi\left(u_{0}, E\right)}=\left.\frac{d}{d u} \log \psi(u, E)\right|_{u=u_{0}}
$$

which follows since $\psi\left(u_{0}, E\right)=1$ and $\psi^{\prime}\left(u_{0}, E\right)=m\left(u_{0}, E\right)$. The different $m$-functions for different $\alpha$ are related by linear fractional transformations, namely

$$
m_{\alpha}\left(u_{0}, E\right)=\frac{(\cos \alpha) m_{0}\left(u_{0}, E\right)-\sin \alpha}{(\sin \alpha) m_{0}\left(u_{0}, E\right)+\cos \alpha},
$$

the principal $m$-function determines them all. With our definition the $m$-function maps the upper half-plane $\mathbb{C}^{+}=\{E: \operatorname{Im}(E)>0\}$ into itself, so that it is a Herglotz function. (Our definition is the negative of the $m$-function for $\alpha$ given in [67, Section 2.1], which maps the upper halfplane to the lower half-plane $\mathbb{C}^{-}$, cf. [67, Section 3.1].) As noted above, in the general case an $m$-function need not analytically continue across the real axis anywhere. However in the case at hand, that of pure discrete spectrum, the Weyl-Titchmarsh $m$-function extends to a meromorphic function on $\mathbb{C}$, and all of its singularities are simple poles, located on the real axis. This 
case is treated in Chapter II of Titchmarsh [67]. The principal $m$-function $m\left(u_{0}, E\right)$ then has a pole at any point where $\psi\left(u_{0}, E\right)$ would be a multiple of $\varphi\left(u_{0}, E\right)$.

Theorem B.1. The Schrödinger equation on the half-line $\left[u_{0}, \infty\right)$ with Morse potential $V_{k}(u)=\frac{1}{4} \mathrm{e}^{2 u}+k \mathrm{e}^{u}$ has principal Weyl-Titchmarsh $m$-function $m\left(u_{0}, E\right)$ with eigenvalue $E=z^{2}$ for endpoint $u_{0}$ given by

$$
m\left(u_{0}, z^{2}\right)=-\frac{W_{1-k, \mathrm{i} z}\left(\mathrm{e}^{u_{0}}\right)}{W_{-k, \mathrm{i} z}\left(\mathrm{e}^{u_{0}}\right)}+\left(\frac{1}{2} \mathrm{e}^{u_{0}}+k-\frac{1}{2}\right) .
$$

Proof. Since the Morse potential on the half-line $\left[u_{0}, \infty\right)$ has pure discrete spectrum, the $m$-function will be a meromorphic function of the variable $E=z^{2}$. Theorem 2.1(1) shows that for $\Im(E)>0$ the subordinate solution is given by

$$
\psi\left(u, z^{2}\right):=\mathrm{e}^{-\frac{u}{2}} W_{-k, \mathrm{i} z}\left(\mathrm{e}^{u}\right)=\mathrm{e}^{-\frac{u}{2}} W_{-k,-\mathrm{i} z}\left(\mathrm{e}^{u}\right)
$$

We conclude that

$$
\begin{aligned}
m\left(u, z^{2}\right) & =\frac{\psi^{\prime}\left(u, z^{2}\right)}{\psi\left(u, z^{2}\right)} \\
& =-\frac{1}{2}+\mathrm{e}^{u} \frac{W_{-k, \mathrm{i} z}^{\prime}\left(\mathrm{e}^{u}\right)}{W_{-k, \mathrm{i} z}\left(\mathrm{e}^{u}\right)}
\end{aligned}
$$

in which $W_{\kappa, \mathrm{i} z}^{\prime}(x)=\frac{d}{d x} W_{\kappa, \mathrm{i} z}(x)$. We now use the identity $[1,13.4 .33]$

$$
x W_{\kappa, \mu}^{\prime}(x)=\left(\frac{1}{2} x-\kappa\right) W_{\kappa, \mu}(x)-W_{\kappa+1, \mu}(x) .
$$

Taking $x=\mathrm{e}^{u}$ and substituting this in (B.5) results in

$$
m\left(u, z^{2}\right)=\left(\frac{1}{2} \mathrm{e}^{u}+k-\frac{1}{2}\right)-\frac{W_{1-\mathrm{i} z, \mu}\left(\mathrm{e}^{u}\right)}{W_{-k, \mathrm{i} z}\left(\mathrm{e}^{u}\right)},
$$

the desired formula.

Remarks. (1) The principal $m$-function contains enough information to uniquely reconstruct the potential $V(u)$ on $\left[u_{0}, \infty\right)$, under very general conditions; this is a particular kind of inverse spectral problem. In the case where the Schrödinger operator has pure discrete spectrum, this follows from the fact that the Dirichlet problem eigenvalues are the poles of $m\left(u_{0}, E\right)$, and the Neumann problem eigenvalues are the zeros of $m\left(u_{0}, E\right)$; necessarily all 
of these fall on the real axis. The Dirichlet and Neumann spectrum together are known to be enough to determine the spectrum.

(2) An important feature of the principal $m$-function is that it satisfies a Riccati equation in the $u$-variable,

$$
\frac{d}{d u} m(u, E)+m(u, E)^{2}=V(u)-E .
$$

This can be exploited in inverse spectral reconstruction of the potential.

(3) It is well known that the principal $m$-function attached to a Schrödinger operator on a half-line having a potential $V(u)$ that is locally $L^{1}$ (and which is in the limit point case at $\infty$ ) has strong restrictions on its asymptotics in the upper half-plane as $\Im(E) \rightarrow \infty$. Let $E=z^{2}$ with $z$ confined to a sector $0<\epsilon \leq \arg z \leq \frac{\pi}{2}-\epsilon$. Then for $a>0$ and $0 \leq u_{0}<a$, for sufficiently large $|z|$ with $\epsilon<\arg (z)<\frac{\pi}{2}-\epsilon$, there holds

$$
m\left(u_{0}, z^{2}\right)=\mathrm{i} z-\int_{0}^{a-u_{0}} V\left(t+u_{0}\right) \mathrm{e}^{2 \mathrm{i} z t} d t+O\left(\frac{1}{|z|}\right)
$$

where the constant in the $O$-symbol depends on $a$ and $\epsilon$. The basic result asserting $m\left(u_{0}, E\right)$ equals $\mathrm{i} \sqrt{E}$ plus an error term is due to Atkinson [3, Theorem $2 \mathrm{ff}$.], who deduced it using (B.8). Atkinson used a different definition of $m$-function, which in terms of the definition used here is $\frac{-1}{m(u, z)}$. (That is, Atkinson used the Neumann boundary condition in defining his $m$-function, while the principal $m$-function is based on the Dirichlet boundary condition at the left endpoint.) A result in the form (B.9) appears in Rybkin [58, Corollary 5.2]. Combining Theorem B.1 with the asymptotics (B.9) provides information on the relative sizes of two Whittaker functions $W_{1-k, \mu}\left(\mathrm{e}^{u}\right)$ and $W_{-k, \mu}\left(\mathrm{e}^{u}\right)$ for $\mu$ in the second quadrant region of the $\mu$-plane.

\section{Acknowledgments}

Christian Remling supplied helpful comments and references, and J.-F. Burnol made useful comments on his work and on $m$-functions. I thank them and the reviewers for helpful comments and corrections. This work was supported by the NSF under Grants DMS-0500555 and DMS-0801029.

\section{References}

[1] M. Abramowitz and I. A. Stegun, Eds., Handbook of mathematical functions, with formulas, graphs and mathematical tables, National Bureau of Standards, Washington D.C., Seventh Corrected Printing, May 1968. 
[2] F. V. Atkinson, Discrete and continuous boundary problems, Academic Press, Inc., New York, 1964.

[3] F. V. Atkinson, On the location of the Weyl circles, Proc. R. Soc. Edinb. Sect. A 88 (1981), 345-356.

[4] M. V. Berry and J. P. Keating, The Riemann zeros and eigenvalue asymptotics, SIAM Rev. 41 (1999), 236-266.

[5] P. Biane, Matrix valued Brownian motion and a paper by Pólya, Séminaire de Probabilitiées, Springer Lecture Notes in Math., to appear, eprint: arXiv:0811.1490v1

[6] L. de Branges, Self-recipocal functions, J. Math. Anal. Appl. 9 (1964), 433-457.

[7] L. de Branges, Hilbert spaces of entire functions, Englewood Cliffs, NJ, Prentice-Hall, 1968.

[8] H. Buchholz, The confluent hypergeometric function, with special emphasis on its applications, Springer-Verlag, New York, 1969.

[9] J.-F. Burnol, Sur certains espaces de Hilbert de fonctions entieres, lies a la transformation de Fourier et aux fonctions $L$ de Dirichlet et de Riemann, C. R. Acad. Sci. Paris, ser. I, 333 (2001), 201-206.

[10] J.-F. Burnol, Sur les "Espaces de Sonine" associes par de Branges a la transformation de Fourier, C. R. Acad. Sci. Paris Math. 335 (2002), 689-692.

[11] J.-F. Burnol, Des equations de Dirac et de Schrödinger pour la transformation de Fourier, C. R. Acad. Sci. Paris Math. 336 (2003), 919-924.

[12] J.-F. Burnol, Two complete and minimal systems associated with the zeros of the Riemann zeta function, J. Théor. Nombres Bordeaux 16 (2004), 65-94.

[13] J.-F. Burnol, Spacetime causality in the study of the Hankel Transform, Ann. Henri Poincaré 7 (2006), 1013-1034.

[14] J.-F. Burnol, Scattering, determinants, hyperfunctions in relation to $\frac{\Gamma(1-s)}{\Gamma(s)}$, arXiv eprint: math.NT/0602045

[15] J.-F. Burnol, On some bound and scattering states associated with the cosine kernel, eprint: arxiv:0801.0530 v1. 
The Schrödinger operator with Morse potential on the right half-line 357

[16] K. M. Case, Stability of an idealized atmosphere. I. Discussion of results, Phys. Fluids 3 (1960), 149-154.

[17] E. A. Coddington and N. Levinson, Theory of ordinary differential equations, New York, McGraw-Hall, 1955.

[18] A. Connes, Trace formula in noncommutative geometry and the zeros of the Riemann zeta function, Selecta Math. (N. S.) 5 (1999), 29-106.

[19] F. Cooper, A. Khare and U. Sukhatme, Supersymmetry and quantum mechanics, Phys. Rep. 251 (1995), 267-385.

[20] A. Deitmar, A Polya-Hilbert operator for automorphic L-functions, Indag. Math. 12 (2001), 157-175.

[21] L. A. Dikii, On zeros of Whittaker and MacDonald functions with complex index (Russian), Izv. Akad. Nauk SSSR Ser. Mat. 24 (1960), 943954.

[22] L. A. Dikii, On the stability of plane parallel flows of an inhomogeneous fluid, Prikl. Mat. Meh. 24 (1960), 249-257 (Russian). Translated as: J. Appl. Math. Mech. 24 (1960), 357-369.

[23] H. Dym, An introduction to de Branges spaces of entire functions with applications to differential equations of the Sturm-Liouville type, Adv. Math. 5 (1971), 395-471.

[24] H. Dym and H. P. McKean, Gaussian processes, function theory and the inverse spectral problem, New York, Academic Press, 1976.

[25] F. J. Dyson, Stability of an idealized atmosphere II: zeros of the confluent hypergeometric function. Phy. Fluids 3 (1960), 155-157.

[26] H. E. Edwards, Riemann's zeta function, New York, Academic Press, 1974.

[27] A. Erdélyi, W. Magnus, F. Oberhettinger and F. G. Tricomi, Higher transcendental functions, Vol. I, Based on notes left by H. Bateman, McGraw-Hill Book Co., New York, 1953.

[28] A. Erdélyi, W. Magnus, F. Oberhettinger and F. G. Tricomi, Higher transcendental functions, Vol. II, Based on notes left by H. Bateman, McGraw-Hill Book Co., New York, 1953.

[29] W. N. Everitt and H. Kalf, Weyl's work on singular Sturm-Liouville operators, in Groups and Analysis: The Legacy of Hermann Weyl, ed. 
K. Tent, London Math. Soc. Lecture Notes, 354, Cambridge Univ. Press, Cambridge, 2008.

[30] S. Flugge, Practical quantum mechanics, Springer-Verlag, New York, 1974.

[31] G. Gasper, Using integrals of squares of certain real-valued special functions to prove that the Pólya $\Xi^{*}(z)$ function, the functions $K_{\mathrm{i} z}(a), a>0$ and some other entire functions have only real zeros, eprint: arxiv:0801.2996, 19 January 2008.

[32] D. J. Gilbert and D. B. Pearson, On subordinacy and analysis of the spectrum of one-dimensional Schrödinger operators, J. Math. Anal. Appl. 128 (1987), 30-56.

[33] I. S. Gradshteyn and I. M. Ryzhik, Table of integrals, series and products (corrected and revised edition), Academic Press, New York, 1983.

[34] C. Grosche, The path integral on the Poincaré upper half-plane with a magnetic field and the Morse potential, Ann. Phys. (N. Y.) 187 (1988), $110-134$.

[35] N. Ikeda and H. Matsumoto, Brownian motion on the hyperbolic plane and Selberg trace formulae, J. Funct. Anal. 163 (1999), 63-110.

[36] M. E. H. Ismail and E. Koelink, The J-matrix method: a survey of tridiagonalization, eprint: arXiv:0810.4558 v.1.

[37] H. Iwaniec and P. Sarnak, Perspectives on the analytic theory of L-functions, GAFA 2000, Tel Aviv, 1999, Geom. Funct. Anal., 2000, (special volume, Part II), 705-741.

[38] N. Katz and P. Sarnak, Zeros of zeta functions and symmetry, Bull. Amer. Math. Soc. 36 (1999), 1-26.

[39] E. Kamke, Differentialgleichungen, Lösungmethoden und Lösungen, Leipzig, Teubner, 1943.

[40] J. C. Lagarias, Zero spacing distributions for differenced $L$-functions, Acta Arith. 120 (2005), 159-181.

[41] J. C. Lagarias, Hilbert spaces of entire functions and Dirichlet Lfunctions, in: Frontiers in Number Theory, Physics and Geometry I: On Random Matrices, Zeta Functions, and Dynamical Systems, eds. P. E. Cartier, B. Julia, P. Moussa and P. van Hove, Springer-Verlag, Berlin, 2006, 365-377. 
The Schrödinger operator with Morse potential on the right half-line 359

[42] J. C. Lagarias, Hilbert spaces of entire functions and automorphic Lfunctions, in preparation.

[43] J. C. Lagarias, Morse spaces of entire functions, in preparation.

[44] P. D. Lax and R. S. Phillips, Scattering theory for automorphic functions, Annals of Math. Studies, 87, Princeton University Press, Princeton, 1976.

[45] B. M. Levitan and I. S. Sargsjan, Introduction to spectral theory: selfadjoint ordinary differential operators, Amer. Math. Soc. Translations of Math. Monographs, 39, AMS, Providence, RI 1975.

[46] B. Ja. Levin, Distribution of zeros of entire functions, revised edition, American Math. Soc., Providence, RI 1980.

[47] R. Meyer, On a representation of the idele class group related to primes and zeros of L-functions, Duke Math. J. 127 (2005), 519-595.

[48] R. Meyer, A spectral interpretations for the zeros of the Riemann zeta function, Seminars Winter Term 2004/2005, Mathematisches Institut, Georg-August-Universität Göttingen, 117-137, Universitätsdrucke Göttingen, Góttingen, 2005.

[49] A. Milne, On the roots of the confluent hypergeometric functions, Proc. Edinb. Math. Soc. Ser. I, 33 (1915), 48-64.

[50] P. Morse, Diatomic molecules according to the wave mechanics II. Vibrational modes, Phys. Rev. 34 (1929), 57-67.

[51] J. Negro, L. M. Nieto and O. Rosas-Ortiz, Confluent hypergeometric equations and related solvable potentials in quantum mechanics, J. Math. Phys. 41 (12) (2000), 7964-7996.

[52] B. S. Pavlov and L. D. Faddeev, Scattering theory and automorphic functions (Russian), Boundary value problems of mathematical physics and related questions in the theory of functions 6, Zap. Nauk. Sem. Leningrad. Otdel. Mat. Inst . Stekov (LOMI) 27 (1972), 161-193.

[53] G. Polya, Bemerkung Über die Integraldarstellung der Riemannschen

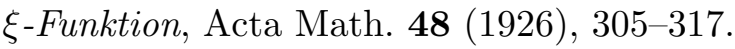

[54] G. Polya, Über trigonometrische Integrale mit nur rellen Nullstellen, J. Reine Angew Math. 158 (1927), 6-18. 
[55] M. Reed and B. Simon, Methods of modern mathematical physics II. Fourier analysis, self-adjointness, Academic Press, New York, 1975.

[56] M. Reed and B. Simon, Methods of modern mathematical physics IV. Analysis of operators, Academic Press, New York, 1978.

[57] C. Remling, Schrödinger operators and de Branges spaces, J. Funct. Anal. 196 (2002), 323-394.

[58] A. Rybkin, Some new and old asymptotic representations of the Jost solution and the Weyl m-function for Schrödinger operators on the line, Bull. London Math. Soc. 34 (2002), 61-72.

[59] G. Sierra, The Riemann zeros and the cyclic renormalization group, J. Stat. Mech. Theory Exp. 2005, (12), P12006, 29pp.

[60] G. Sierra, $H=x p$ with interaction and the zeta zeros, Nucl. Phys. B 776 (2007), 327-364.

[61] G. Sierra, On the quantum reconstruction of the Riemann zeros, J. Phys A: Math Theor. 41 (30), (2008), 304041, 17pp.

[62] G. Sierra, A quantum mechanical model of the Riemann zeros, New J. Phys. 10 (2008), 033016, 32pp.

[63] G. Sierra and P. K. Townsend, Landau levels and Rieman zeros, Phys. Rev. Lett. 101 (11), (2008), 110201, 4pp.

[64] L. J. Slater, Confluent Hypergeometric functions, Cambridge University Press, New York, 1960.

[65] C. Soulé, Sur les zéros des fonctions L automorphes, C. R. Acad. Sci. Paris, Sé. I 328, (1999), 955-958.

[66] E. C. Titchmarsh, Theory of functions, 2nd edn., Oxford University Press, Oxford, 1939 (reprint: 1964).

[67] E. C. Titchmarsh, Eigenfunction expansions associated with second order differential equations, Part I, 2nd edn., Oxford, Clarendon Press, 1962 (first edition, 1946).

[68] E. C. Titchmarsh, The theory of the Riemann zeta function, 2nd edn., Revised by D. R. Heath-Brown, Clarendon Press, Oxford, 1986.

[69] F. G. Tricomi, Über die Abzáhlung der Nullstellen der konfluenten hypergeometrischen Funktionen, Math. Z. 52 (1950), 669-675. 
The Schrödinger operator with Morse potential on the right half-line 361

[70] C. Truesdell, A unified theory of special functions, Ann. Math. Studies, 18, Princeton University Press, Princeton, 1948.

[71] G. E. Tvestkoff, On roots of Whittaker's functions, C. R. (Doklady) Acad. Sci. URSS (N. S.) 32 (1941), 10-12 (Russian).

[72] Y. Uetake, Lax-Phillips scattering for automorphic functions based on the Eisenstein transform, Integral Equations Operator Theory 60 (2008), 271-283.

[73] E. T. Whittaker, An expression of certain known functions as generalized hypergeometric functions, Bull. Amer. Math. Soc. 10 (1904), 125-134.

[74] E. T. Whittaker and G. N. Watson, A course of modern analysis, 4th edn., Cambridge University Press, Cambridge, 1963.

UNiVERSITY OF MichigAN

AnN Arbor, MI 48109-1043, USA

E-mail address: lagarias@umich.edu

Received December 18, 2008 
